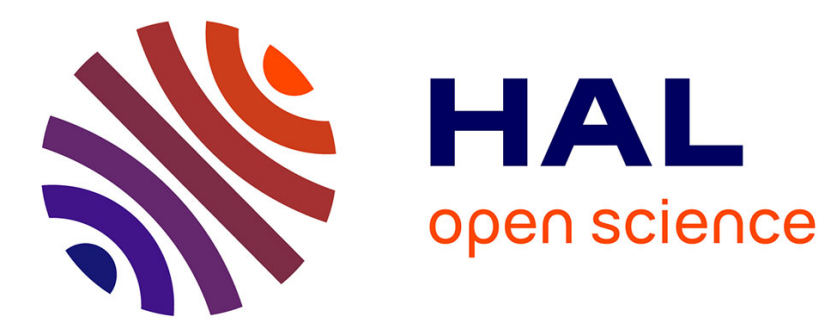

\title{
Parsimonious finite-volume frequency-domain method for 2D P-SV-wave modeling
}

R. Brossier, Jean Virieux, Stéphane Operto

\section{To cite this version:}

R. Brossier, Jean Virieux, Stéphane Operto. Parsimonious finite-volume frequency-domain method for 2D P-SV-wave modeling. Geophysical Journal International, 2008, 175, pp.541-559. 10.1111/j.1365246X.2008.03839.x . insu-00200052v2

\section{HAL Id: insu-00200052}

\section{https://hal-insu.archives-ouvertes.fr/insu-00200052v2}

Submitted on 21 Sep 2009

HAL is a multi-disciplinary open access archive for the deposit and dissemination of scientific research documents, whether they are published or not. The documents may come from teaching and research institutions in France or abroad, or from public or private research centers.
L'archive ouverte pluridisciplinaire HAL, est destinée au dépôt et à la diffusion de documents scientifiques de niveau recherche, publiés ou non, émanant des établissements d'enseignement et de recherche français ou étrangers, des laboratoires publics ou privés. 


\title{
Parsimonious finite-volume frequency-domain method for 2D P-SV-wave modelling
}

\author{
R. Brossier ${ }^{1}$, J. Virieux ${ }^{2}$ and S. Operto ${ }^{3}$ \\ ${ }^{1}$ Géosciences Azur, Université de Nice Sophia-Antipolis, CNRS,IRD, Université Pierre et Marie Curie, \\ Valbonne, France.E-mail: brossier@geoazur.unice.fr. \\ ${ }^{2}$ Laboratoire de Géophysique Interne et Tectonophysique, Université Joseph Fourier, CNRS, Grenoble, France. \\ E-mail: Jean.Virieux@obs.ujf-grenoble.fr. \\ ${ }^{3}$ Géosciences Azur, Université de Nice Sophia-Antipolis, CNRS,IRD, Université Pierre et Marie Curie, \\ Villefranche-sur-mer, France. E-mail: operto@geoazur.obs-vlfr.fr. \\ Contact : R. Brossier, brossier@geoazur.unice.fr, Ph/fax : +33492942662/+33492942610
}

Accepted 2008 April 30. Received 2008 March 5; in original form 2007 December 10

\begin{abstract}
SUMMARY
A new numerical technique for solving 2D elastodynamic equations based on a finite-volume frequency-domain approach is proposed. This method has been developed as a tool to perform two-dimensional (2D) elastic frequency-domain full-waveform inversion. In this context, the system of linear equations that results from the discretisation of the elastodynamic equations is solved with a direct solver, allowing efficient multiple-source simulations at the partial expense of the memory requirement. The discretisation of the finite-volume approach is through triangles. Only fluxes with the required quantities are shared between the cells, relaxing the meshing conditions, as compared to finite-element methods. The free surface is described along the edges of the triangles, which can have different slopes. By applying a parsimonious strategy, the stress components are eliminated from the discrete equations and only the velocities are left as unknowns in the triangles. Together with the local support of the P0 finite-volume stencil, the parsimonious approach allows the minimising of core memory requirements for the simulation. Efficient perfectly matched layer absorbing conditions have been designed for damping the waves around the grid. The numerical dispersion of this FV formulation is similar to that of $O\left(\Delta x^{2}\right)$ staggered-grid finite-difference formulations when considering structured triangular meshes. The validation has been performed with analytical solutions of several canonical problems and with numerical solutions computed with a well-established finite-difference time-domain method in heterogeneous media. In the presence of a free surface, the finite-volume method requires ten triangles per wavelength for a flat topography, and fifteen triangles per wavelength for more complex shapes, well below the criteria required by the staircase approximation of $O\left(\Delta x^{2}\right)$ finite-difference methods. Comparisons between the frequency-domain finite-volume and the $O\left(\Delta x^{2}\right)$ rotated finite-difference methods also show that the former is faster and less memory demanding for a given accuracy level, an attractive feature for frequency-domain seismic inversion. We have thus developed an efficient method for 2D P-SV-wave modelling on structured triangular meshes as a tool for frequency-domain full-waveform inversion. Further work is required to improve the accuracy of the method on unstructured meshes.
\end{abstract}

Key words: seismic wave propagation, numerical modelling, finite volume approach, elastic waves, frequency domain

\section{INTRODUCTION}

Seismic wave propagation has been investigated by various numerical methods, such as finite-difference (FD), finite-element (FE) and boundary integral equations. When considering media with complex topography and a possible water layer, few approaches are available for efficient forward modelling, especially when the modelling scheme is dedicated to seismic full-waveform inversion applications that require thousands of forward modelling stages. The FD staggered-grid method proposed by Madariaga (1976) and Virieux (1986a) using the Yee 


\section{Brossier \& al}

scheme (Yee, 1966), which is based on a first-order velocity-stress hyperbolic system, is relatively popular and has been used intensively in the time domain for seismic imaging in spite of the limited solution accuracy when considering free surfaces with topography (Gauthier et al., 1986). The solution has been shown to be stable when considering fluid zones inside the FD grid. The staircase approximation of the free surface, as proposed by Robertsson (1996), requires dense meshing. Recently, attempts have been proposed through mesh deformation (Hestholm and Ruud, 2002) and through immersed implicit boundary methods (Lombard et al., 2008), although these need specific numerical developments near to the free surface.

The FE approaches, as proposed for elastic wave propagation by Marfurt (1984), have been reshaped recently with low-order interpolation for efficient 3D simulations (Bielak et al., 2003; Yoshimura et al., 2003; Koketsu et al., 2004), while recent high-order interpolations have led to the so-called spectral element method (Faccioli et al., 1997; Vilotte et al., 2005; Komatitsch and Vilotte, 1998; Chaljub et al., 2003). Both of these approaches with different mesh densities have allowed accurate modelling of free surface effects, as these FE methods adapt the mesh to the surface topography. Moczo et al. (1997) have proposed the combination of FD and FE methods to deal efficiently with complex topographies. Due to the weak formulation of these methods, the fluid/solid interface has to be tackled by explicit boundary conditions.

Other numerical methods have tried to avoid the necessary continuity of fields at shared nodes between elements, which has led to unconventional FE methods (Casadei et al., 2002) and to discontinuous Galerkin (DG) methods (Cockburn et al., 2000), which have been popularised in seismology by Dumbser, Käser and co-workers (Käser and Dumbser, 2006; Dumbser and Käser, 2006; Käser et al., 2007; de la Puente et al., 2007; Dumbser et al., 2007). Both methods are particularly demanding of computer resources. Explicit boundary conditions have been worked out for fracture problems by BenJemaa et al. (2007) for a low-order interpolation of the DG method, which is nothing more than a FV method with constant value interpolation inside each element. These FV/DG methods work in the time domain using both velocity and stress fields, with very promising perspectives.

The aim of this study is to develop a frequency-domain modelling method that is suited to seismic imaging applications performed by frequency-domain full-waveform inversion. The above-mentioned methods may not be the optimal ones for this kind of application, for the reasons explained below. With the success of full-waveform inversion in the frequency domain (Pratt and Worthington, 1990; Pratt et al., 1996, 1998), applications to real data using the acoustic approximation for 2D geometries have been performed for imaging complex structures (Ravaut et al., 2004; Operto et al., 2006), while the reconstruction of elastic parameters has been found to be a quite challenging problem (Gelis et al., 2007). These approaches are based on a hierarchical multiscale inversion scheme that proceeds over a coarse subset of frequencies, from the low frequencies to the higher ones, and that requires a large number of forward simulations at each iteration of the multiscale reconstruction. To consider both onshore and offshore applications of full-waveform inversion, the modelling method must be accurate in the case of complex media that incorporate either a free surface of arbitrary shape or a water layer, while remaining sufficiently fast to be able to perform multiple simulations in a tractable time. High-order accuracy methods that might perform well in the time domain turn out to be prohibitive in the frequency domain if the linear system that results from the discretisation of the frequency-domain wave equation is solved with a direct solver (Stekl and Pratt, 1998; Hustedt et al., 2004). Direct solvers are generally used to perform 2D frequency-domain wave modelling because solutions for multiple sources can be efficiently computed by substitutions once the impedance matrix has been LU-factorized. A key feature for numerical efficiency is the compactness of the spatial operator that controls the numerical bandwidth of the impedance matrix, and therefore its fill-in during factorization. Therefore, we shall concentrate our comparisons on $O\left(\Delta x^{2}\right)$ FD methods that minimise the memory requirement of the linear system resolution for the frequency-domain formulation. The $O\left(\Delta x^{4}\right)$ FD method (Levander, 1988) has been shown to be an efficient compromise between memory saving and CPU demand for time-domain formulation. Unfortunately, even with a coarser meshing, this higher-order stencil dramatically increases the memory cost of the linear system resolution in the frequency-domain formulation (Hustedt et al., 2004).

Optimal compact FD stencils based on the so-called mixed-grid method that combines the Cartesian FD stencil and the rotated FD stencil (Saenger et al., 2000) and anti-lumped mass features have been designed for frequency-domain wave-propagation modelling. This approach of combining stencils has been shown to be very efficient for the acoustic wave equation (Jo et al., 1996; Hustedt et al., 2004), for which only four grid points per wavelength can be used. Stekl and Pratt (1998) have shown limitations in the elastic case, when a liquid-solid interface is involved; here, the Cartesian stencil has to be removed. The same limitation applies when considering a complex free surface, leaving second-order FD stencils. For the rotated FD stencil (Saenger et al., 2000), the free surface condition is verified simply through a vacuum approach, with a minimum of 25 nodes per shear wavelength for a flat topography, and up to 60 nodes per shear wavelength with a complex topography (Saenger and Bohlen, 2004; Bohlen and Saenger, 2006) in the time domain. Gelis et al. (2007) have also shown that this is similar in the frequency domain, leading to the solving of rather significant sparse linear systems. The method we propose here will significantly reduce this dense sampling near to the free surface, a key issue for frequency formulation.

We thus present a FV $P_{0}$ method based on a first-order hyperbolic elastodynamic system in the frequency domain, as is usual for FV formulations (Remaki, 2000). We will deduce the discretised system of linear equations to be solved only for velocity components, using the parsimonious strategy (Luo and Schuster, 1990). The numerical dispersion behaviour of this scheme will be analysed before discussing the perfectly matched layer (PML) conditions (Berenger, 1994). The implementation of the source will require specific attention before going on to numerical validations against both the analytical solutions and the numerical solutions obtained by other numerical methods. For these examples, we will show the respective numerical costs of frequency-domain FV and $O\left(\Delta x^{2}\right)$ FD methods. We will conclude with the potential of this FV approach in the frequency domain for the modelling of seismic 2D P-SV waves and the perspectives for inversion. 


\section{FINITE VOLUME FORMULATION IN THE FREQUENCY DOMAIN}

We consider a first-order hyperbolic elastodynamic system for 2D P-SV waves in isotropic medium in the frequency domain where both velocities $\left(V_{x}, V_{z}\right)$ and stress $\left(\sigma_{x x}, \sigma_{z z}, \sigma_{x z}\right)$ are unknown quantities as described by the following differential system :

$$
\begin{aligned}
-\iota \omega V_{x} & =\frac{1}{\rho(\mathbf{x})}\left\{\frac{\partial \sigma_{x x}}{\partial x}+\frac{\partial \sigma_{x z}}{\partial z}\right\}+F_{x} \\
-\iota \omega V_{z} & =\frac{1}{\rho(\mathbf{x})}\left\{\frac{\partial \sigma_{x z}}{\partial x}+\frac{\partial \sigma_{z z}}{\partial z}\right\}+F_{z} \\
-\iota \omega \sigma_{x x} & =(\lambda(\mathbf{x})+2 \mu(\mathbf{x})) \frac{\partial V_{x}}{\partial x}+\lambda(\mathbf{x}) \frac{\partial V_{z}}{\partial z}-\iota \omega \sigma_{x x_{0}} \\
-\iota \omega \sigma_{z z} & =\lambda(\mathbf{x}) \frac{\partial V_{x}}{\partial x}+(\lambda(\mathbf{x})+2 \mu(\mathbf{x})) \frac{\partial V_{z}}{\partial z}-\iota \omega \sigma_{z z_{0}} \\
-\iota \omega \sigma_{x z} & =\mu(\mathbf{x})\left\{\frac{\partial V_{x}}{\partial z}+\frac{\partial V_{z}}{\partial x}\right\}-\iota \omega \sigma_{x z_{0}},
\end{aligned}
$$

where the Lamé coefficients that describe the medium are denoted by $\lambda, \mu$, the density by $\rho$ and the angular frequency by $\omega$. Source terms are either point forces $\left(F_{x}, F_{z}\right)$ or applied stresses $\left(\sigma_{x x_{0}}, \sigma_{z z_{0}}, \sigma_{x z_{0}}\right)$ as introduced in system (1). The pure imaginary number defined as $x^{2}=-1$ is denoted as $\iota$. The Fourier transform follows the usual convention as $f(\omega)=\int_{-\infty}^{+\infty} f(t) e^{-\iota \omega} d t$. To develop a pseudoconservative formulation that will be useful for integration over a surface in $2 \mathrm{D}$, we will consider the following new vector with three components $\vec{T}^{t}=\left(T_{1}, T_{2}, T_{3}\right)=\left(\left(\sigma_{x x}+\sigma_{z z}\right) / 2,\left(\sigma_{x x}-\sigma_{z z}\right) / 2, \sigma_{x z}\right)$. Moreover, we must consider a finite domain, and therefore we apply PML absorbing conditions (Berenger, 1994) through the functions $s_{x}, s_{z}$ for the velocity equations and the functions $s_{x}^{\prime}, s_{z}^{\prime}$ for the stress equations. More details on the expression of these damping functions $s_{x}, s_{z}, s_{x}^{\prime}, s_{z}^{\prime}$ are given below. The new differential system equivalent to system (1) can be written as :

$$
\begin{aligned}
-\iota \omega \rho V_{x} & =s_{x} \frac{\partial\left(T_{1}+T_{2}\right)}{\partial x}+s_{z} \frac{T_{3}}{\partial z}+\rho F_{x} \\
-\iota \omega \rho V_{z} & =s_{x} \frac{\partial T_{3}}{\partial x}+s_{z} \frac{\partial\left(T_{1}-T_{2}\right)}{\partial z}+\rho F_{z} \\
\frac{-\iota \omega T_{1}}{\lambda+\mu} & =s_{x}^{\prime} \frac{\partial V_{x}}{\partial x}+s_{z}^{\prime} \frac{\partial V_{z}}{\partial z}-\frac{\iota \omega T_{1}^{0}}{\lambda+\mu} \\
\frac{-\iota \omega T_{2}}{\mu} & =s_{x}^{\prime} \frac{\partial V_{x}}{\partial x}-s_{z}^{\prime} \frac{\partial V_{z}}{\partial z}-\frac{\iota \omega T_{2}^{0}}{\mu} \\
\frac{-\iota \omega T_{3}}{\mu} & =s_{x}^{\prime} \frac{\partial V_{z}}{\partial x}+s_{z}^{\prime} \frac{\partial V_{x}}{\partial z}-\frac{\iota \omega T_{3}^{0}}{\mu}
\end{aligned}
$$

We apply a surface integration over a control cell identified by the index $i$. For practical reasons of meshing, control cells are often taken as triangles, but the formulation still stands for any polygonal cells as quadrangles, for example. The geometrical description of a medium depends on the meshing tool we are using, and the filling of 2D space with triangles or 3D space with tetrahedra is often provided by mesh designers. Appendix A illustrates the development of the FV method in a regular Cartesian mesh, which is equivalent to a second-order FD formulation in a Cartesian grid without the staggered grid structure. Therefore, we can compare the numerical performances of the FV method we have developed and the staggered-grid $O\left(\Delta x^{2}\right)$ FD method, which is less intensive than the $O\left(\Delta x^{2}\right)$ FD method considering all of the unknowns at each node of the grid. The formulation presented here is based on triangular cells in a conformal mesh, which imposes three edges and neighbours for each cell considered. The quantities are constant inside each cell, an assumption known as the $P_{0}$ approximation. Higher-order interpolations of $P_{k}$ are often referred to as discontinuous Galerkin methods (Käser and Dumbser, 2006), which should be compared with the new high-order FD schemes, a task that we have not tackled here since these approaches cannot be adapted to our frequency-domain formulation as tackled with a direct solver. According to Green's theorem, we end up with the discrete system written in a vectorial form (see Appendix B for the complete derivation) :

$$
\begin{aligned}
-\iota \omega A_{i} \rho_{i} \vec{V}_{i} & =\sum_{j \in \partial K_{i}} l_{i j} G_{i j}+A_{i} \rho_{i} \vec{F}_{i} \\
-\iota \omega A_{i} \Lambda_{i} \vec{T}_{i} & =\sum_{j \in \partial K_{i}} l_{i j} H_{i j}-\iota \omega A_{i} \Lambda_{i} \vec{T}^{0}{ }_{i}
\end{aligned}
$$

The surface of the $i$ cell is denoted by $A_{i}=\int_{K_{i}} d S$. The index $j \in \partial K_{i}$ labels the three neighbouring cells that have a joint edge with the $i$ cell. The length of the edge between cells $i$ and $j$ is denoted by $l_{i j}$. The numerical approximation of fluxes is denoted by $l_{i j} H_{i j}$ and $l_{i j} G_{i j}$. The matrix $\Lambda_{i}$ is the diagonal matrix defined by $\Lambda_{i}=\operatorname{diag}\left(1 /\left(\lambda_{i}+\mu_{i}\right), 1 / \mu_{i}, 1 / \mu_{i}\right)$. Finally, source vectors applied inside the $i$ cell are denoted by $\vec{F}_{i}$ and $\vec{T}_{i}$.

Centred numerical fluxes of the velocity and stress components between two cells are introduced because they preserve a discrete energy inside the entire zone away from the PML. These were first proposed by Remaki (2000) and used by BenJemaa et al. (2007) for the elastodynamics in the time domain, and Dolean et al. (2006) for Maxwell equations in the frequency domain. This gives us the following 


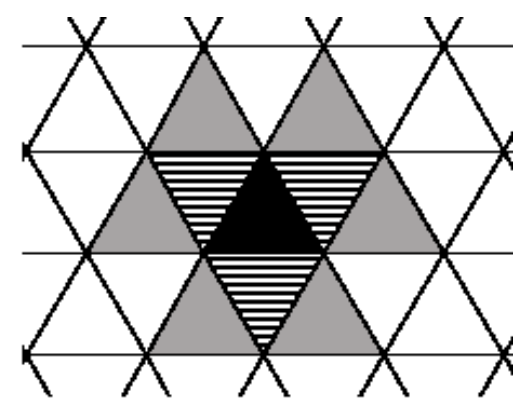

Figure 1. Illustration of the numerical scheme. In black, the central cell for which the solution is computed. The neighbouring cells (hatched) have unknowns that do not influence the scheme. In grey, the neighbours of these neighbouring cells with unknowns that are involved.

estimation of the quantities $G_{i j}$ and $H_{i j}$ :

$$
\begin{aligned}
G_{i j} & =\sum_{r \in\{x, z\}} n_{i j_{r}} N_{k} \frac{s_{r_{i}} \vec{T}_{i}+s_{r_{j}} \vec{T}_{j}}{2} \\
H_{i j} & =\sum_{r \in\{x, z\}} n_{i j_{r}} M_{k} \frac{s_{r_{i}}^{\prime} \vec{V}_{i}+s_{r_{j}}^{\prime} \vec{V}_{j}}{2},
\end{aligned}
$$

where the normal vector component $r$ is oriented for each edge of cell $i$ towards cell $j$ and is denoted by $n_{i j_{r}}$. Projector matrices defined for a vectorial formulation are denoted by $M_{k}$ and $N_{k}$.

The geometrical properties of triangles give

$$
\sum_{j \in \partial K_{i}} l_{i j} P_{i j}=0
$$

where $P_{i j}=\sum_{r \in\{x, z\}} n_{i j_{r}}$. These geometrical properties ensure that for the first-order system, the unknowns in a given cell $i$ depend only on the unknowns of the surrounding cells, and not on the unknowns of the cell $i$ that are cancelled out by the construction as it is built by centred fluxes.

Discrete equations expressing the stress components, as the second vectorial equation of system (2), can be eliminated by inserting them into the fluxes that are required in the velocity equations, the first vectorial equation of system (2), thus reducing the memory requirement for wave propagation. Luo and Schuster (1990) proposed this parsimonious strategy for the FD method, and it turns out that it also works well for this FV method. After elimination of the stress components, we end up with the following two algebraic equations for the two unknown velocity components:

$$
\begin{aligned}
\omega^{2} V_{x_{i}} & =\iota \omega F_{x_{i}}+\frac{\iota \omega}{A_{i} \rho_{i}} \sum_{j \in \partial K_{i}} \frac{l_{i j}}{2}\left\{n_{i j_{x}} s_{x_{j}}\left[\frac{\iota\left(\lambda_{j}+\mu_{j}\right)}{\omega A_{j}} \sum_{k \in \partial K_{j}} \frac{l_{j k}}{2}\left(n_{j k_{x}} s_{x_{k}}^{\prime} V_{x_{k}}+n_{j k_{z}} s_{z_{k}}^{\prime} V_{z_{k}}\right)+T_{1_{j}}^{0}\right]\right. \\
& +n_{i j_{x}} s_{x_{j}}\left[\frac{\iota \mu_{j}}{\omega A_{j}} \sum_{k \in \partial K_{j}} \frac{l_{j k}}{2}\left(n_{j k_{x}} s_{x_{k}}^{\prime} V_{x_{k}}-n_{j k_{z}} s_{z_{k}}^{\prime} V_{z_{k}}\right)+T_{2_{j}}^{0}\right] \\
& \left.+n_{i j_{z}} s_{z_{j}}\left[\frac{\iota \mu_{j}}{\omega A_{j}} \sum_{k \in \partial K_{j}} \frac{l_{j k}}{2}\left(n_{j k_{x}} s_{x_{k}}^{\prime} V_{z_{k}}+n_{j k_{z}} s_{z_{k}}^{\prime} V_{x_{k}}\right)+T_{3_{j}}^{0}\right]\right\} \\
\omega^{2} V_{z_{i}} & =\iota \omega F_{z_{i}}+\frac{\iota \omega}{A_{i} \rho_{i}} \sum_{j \in \partial K_{i}} \frac{l_{i j}}{2}\left\{n_{i j_{z}} s_{z_{j}}\left[\frac{\iota\left(\lambda_{j}+\mu_{j}\right)}{\omega A_{j}} \sum_{k \in \partial K_{j}} \frac{l_{j k}}{2}\left(n_{j k_{x}} s_{x_{k}}^{\prime} V_{x_{k}}+n_{j k_{z}} s_{z_{k}}^{\prime} V_{z_{k}}\right)+T_{1_{j}}^{0}\right]\right. \\
& -n_{i j_{z}} s_{z_{j}}\left[\frac{\iota \mu_{j}}{\omega A_{j}} \sum_{k \in \partial K_{j}} \frac{l_{j k}}{2}\left(n_{j k_{x}} s_{x_{k}}^{\prime} V_{x_{k}}-n_{j k_{z}} s_{z_{k}}^{\prime} V_{z_{k}}\right)+T_{2_{j}}^{0}\right] \\
& \left.+n_{i j_{x}} s_{x_{j}}\left[\frac{\iota \mu_{j}}{\omega A_{j}} \sum_{k \in \partial K_{j}} \frac{l_{j k}}{2}\left(n_{j k_{x}} s_{x_{k}}^{\prime} V_{z_{k}}+n_{j k_{z}} s_{z_{k}}^{\prime} V_{x_{k}}\right)+T_{3_{j}}^{0}\right]\right\},
\end{aligned}
$$

where $k \in \partial K_{j}$ labels the index of the cells $j$, the neighbours of the cell $i$. Due to properties of the triangle given by expression (4), and due to the centred flux estimations and the parsimonious formulation that lead to this algebraic system (5), the velocity unknowns only depend on the velocities of the neighbours of the neighbours of the cell: neighbouring velocity unknowns are not directly involved in the numerical scheme for a given element. Figure 1 illustrates this configuration on a regular mesh, where the numerical scheme centred on the black central cell depends on the unknowns at this cell and on the unknowns belonging to the neighbours of the neighbouring cells (grey cells). No dependency with hatched-cell unknowns is seen, as previously noted by LeVeque (2007) as the black/red pattern of the centred numerical schemes. 

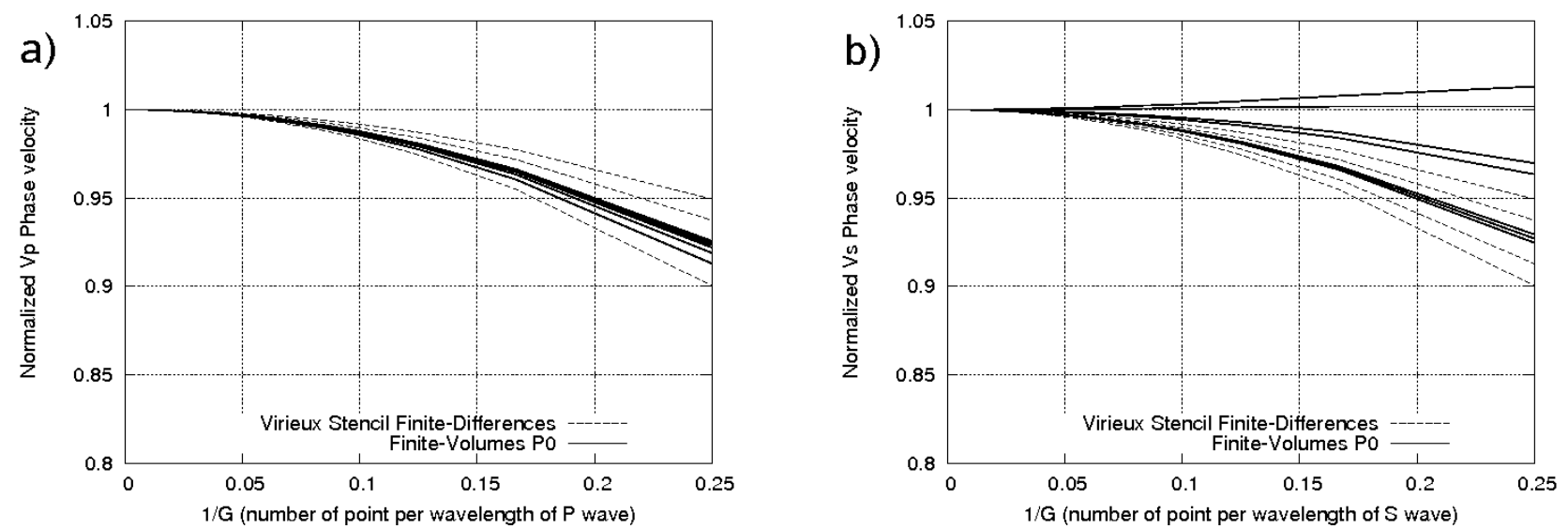

Figure 2. (a) P-wave and (b) S-wave normalised phase-velocity dispersion curves for different plane waves with various incident angles for both the FV (continuous lines) and the $O\left(\Delta x^{2}\right)$ Cartesian FD approaches (dashed lines).

Equations (5) can be recast in matrix form as $\mathcal{A} V=\mathcal{B}$, where the sparse impedance matrix $\mathcal{A}$ contains 14 non-zero coefficients per row in the general case (i.e., without any regular structure), due to the expected irregular numbering of the cells inside the mesh. We should also stress that the corresponding matrix for the parsimonious $O\left(\Delta x^{2}\right)$ rotated FD stencil (Gelis et al., 2007) has 18 non-zero elements.

The free-surface condition is explicitly expressed in the numerical scheme by considering a ghost cell above the free surface that has the same velocity and the opposite stress components to those below the free surface, in order to fulfil the zero stress at the free surface while keeping the correct numerical estimation of the particle velocity at the free surface. Using these velocities and stresses in the ghost cell, the stress flux across the free surface interface vanishes, while the velocity flux is twice the value that would have been obtained by neglecting the flux contribution above the free surface. This boundary condition has been implemented by modifying the impedance matrix accordingly without introducing any new unknown quantities. Similarly, fluid-solid interface modelling requires discontinuities of particle tangential velocities at the boundary. The specific interface conditions that follow the same strategy as that described by BenJemaa et al. (2007) for crack simulations, have also led to a modification of the impedance matrix for both cells that share an interface segment, without increasing its complexity. The same equations with identical numerical schemes are used for both fluid and solid media, where the $\mu$ value is set to zero inside the fluid medium.

\section{NUMERICAL PROPERTIES}

Discretisation leads to numerical dispersion in the particle velocity wavefields. For unstructured meshes, the dispersion could not be estimated analytically, while a regular distribution of equilateral triangles will allow such an investigation. Moreover, we must consider specific properties at the edges of the grid for the extension of the medium to infinity. Finally, the source implementation has to excite the entire grid without exciting a specific sub-grid that is related to the centred pattern of our system.

\subsection{Numerical dispersion analysis}

The numerical dispersion can be estimated for such a discrete system of a regular distribution of equilateral triangles (see the mesh configuration in Figure 1 for this pattern). We here consider an incident-plane wave propagating inside an infinite and homogeneous medium away from the PML and source zones. Due to the regular mesh, the Hermitian structure of the matrix makes eigenvalues real. They are computed numerically for different Poisson ratios from 0.0 to 0.5 , for different number of cells per wavelength, and with incidence angles ranging between $0^{\circ}$ and $180^{\circ}$ with steps of $15^{\circ}$. The dispersion curves are quite similar to those obtained by Virieux (1986b) using an accurate $O\left(\Delta x^{2}\right)$ FD scheme, as shown in Figure 2. The rule of thumb of ten grid points per wavelength appears to provide acceptable propagation dispersion, whatever the value of the shear wave velocity, which can decrease to zero without any numerical instability. The $O\left(\Delta x^{2}\right)$ rotated staggered-grid FD stencil provides similar results with a grid length that is higher by a factor of $\sqrt{2}$ (Saenger et al., 2000). We should also note that we have considered the triangle edge length for the FV approach and the grid step length for the FD approach, as our discrete reference values.

\subsection{PML absorbing boundary conditions}

The frequency domain allows the straightforward numerical implementation of PML conditions, without any splitting of particle velocity components or additional integration of memory variables, as for the time-domain formulation, due to the complex coordinate change (Chew and Liu, 1996). Numerical tests show that the PML efficiency strongly depends on the mesh structure in the PMLs. The PML absorbing 


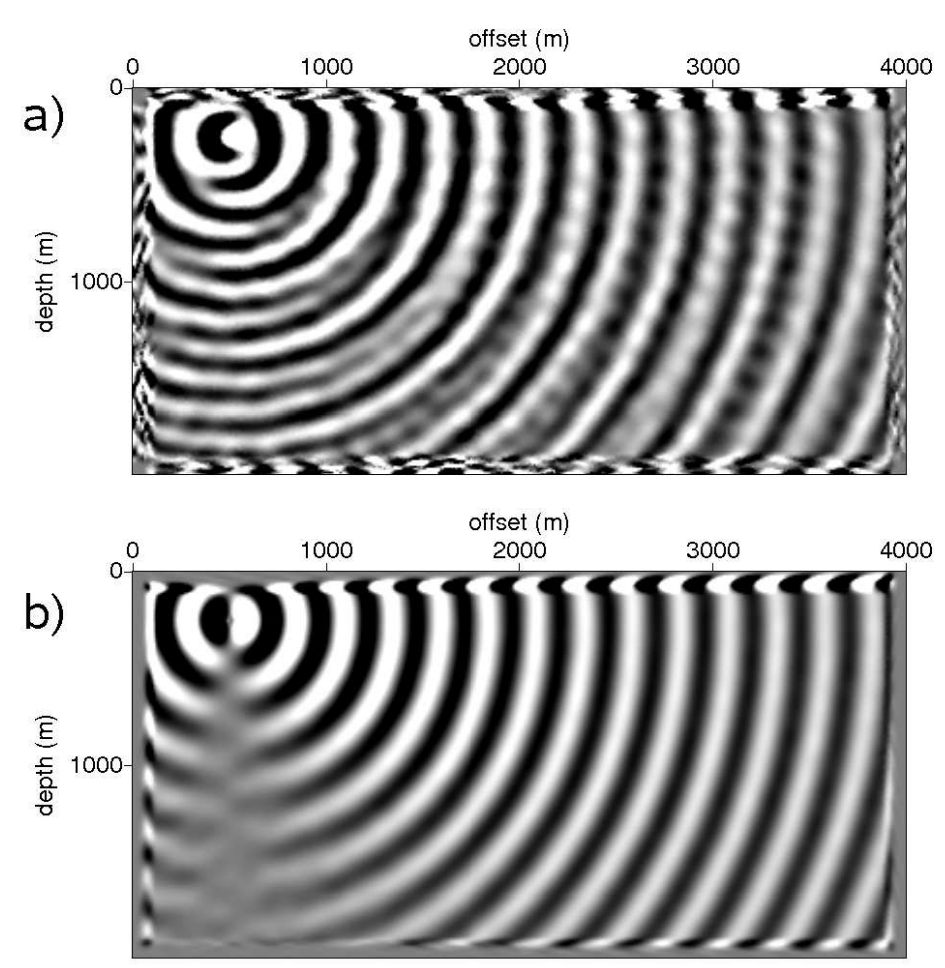

Figure 3. Frequency map solutions where the real part of the horizontal velocity for different PML configurations is displayed. (a) No mesh constraints applied, and (b) with mesh constraints applied.

boundary condition requires that PML-PML interfaces be oriented along the Cartesian directions (Berenger, 1994). This condition is not satisfied if triangles of arbitrary orientations are used in the PML layers. In such a case, we saw poor absorption, as illustrated in Figure 3-(a) for a distribution of non-constrained triangles in arbitrary orientations in the PML layers. Therefore, a constrained mesh in PML zones with multiple layer structures parallel to the Cartesian directions (see Figure 4 for the discretisation of the lower left quarter of the medium) provides efficient absorption of the elastic waves. A transition is, of course, performed between the main central zone of the grid and the PML constrained zones. With this constraint on the mesh construction, at each parallel interface of the PML layers, the major part of the energy is contained along the damped direction, and the numerical flux energy is globally damped by the variations in the PML functions $s_{x}, s_{z}, s_{x}^{\prime}, s_{z}^{\prime}$ as we move deeper into the PML zone. We can here note that use in the PML of quadrangle cells oriented along the Cartesian axes should provide a very efficient behaviour as absorbing boundaries, although this is less easy to implement with triangular mesh generators.

The standard frequency PML function is defined by:

$s_{r}=\frac{1}{1+\iota \gamma_{r} / \omega}$,

where the index $r$ can be $x$ or $z$. A similar expression is obtained for the $s_{r}^{\prime}$ function. Both of these functions are only used inside the PML zones. Outside of the damping zone, the values of the $s_{r}$ and $s_{r}^{\prime}$ functions are simply equal to 1 . The value $\gamma_{r}$ is typically used as a polynomial or cosine function for progressive energy damping. Numerical tests have shown better behaviour with a modified PML function derived from the developments indicated by Drossaert and Giannopoulos (2007). The definition of the damping functions $s_{r}$ and $s_{r}^{\prime}$ are extended through the following equation:

$s_{r}=\frac{1}{\kappa_{r}+\iota \gamma_{r} / \omega}$,

with the expressions $\kappa_{r}$ and $\gamma_{r}$ as cosine functions. A linear dependency of the term $\gamma_{r}$ with frequency has shown good damping behaviour that is almost independent of the frequency. These functions are defined as:

$\begin{aligned} \gamma_{r}(l) & =\omega B \cos \left(\frac{l \pi}{2 l_{p m l}}\right) \\ \kappa_{r}(l) & =1+C \cos \left(\frac{l \pi}{2 l_{p m l}}\right),\end{aligned}$

along the perpendicular direction, where $l_{p m l}$ is the size of the PML zone, which is taken as fifteen cells in the examples that we have selected 


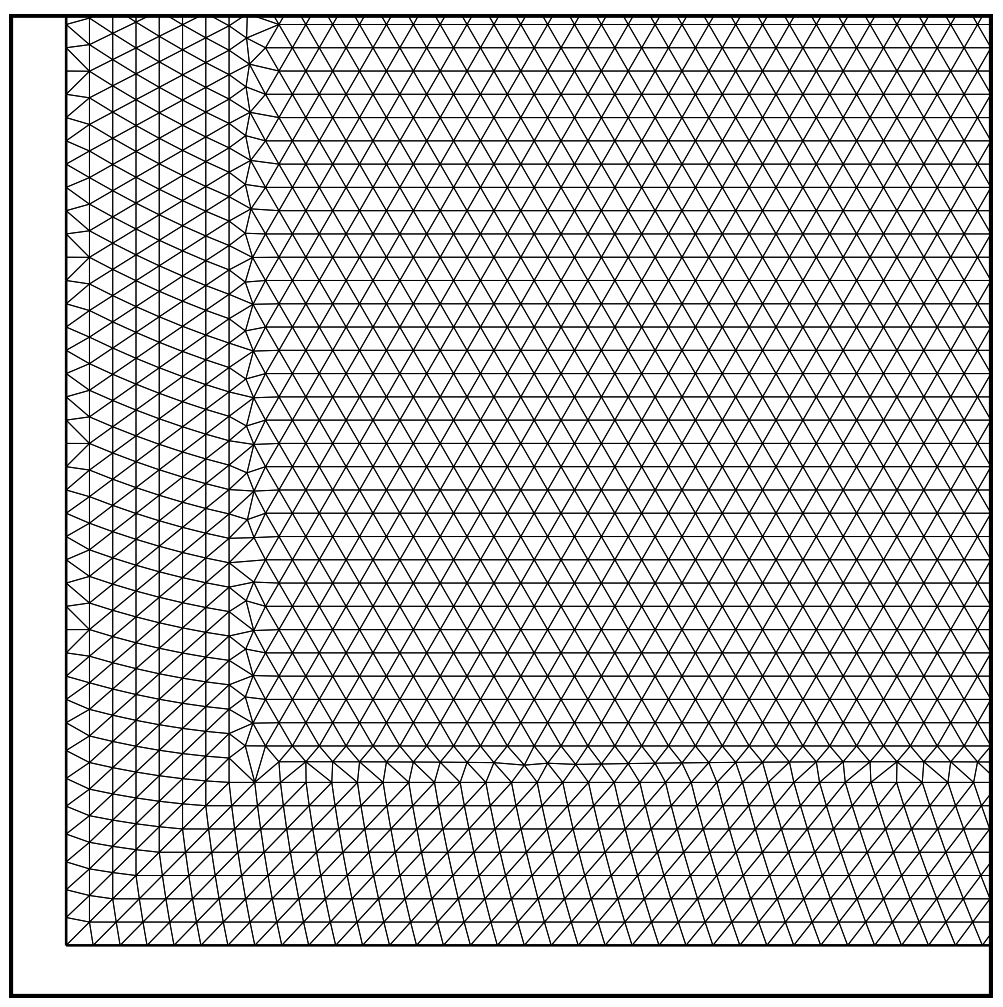

Figure 4. PML construction with multiple layer structures parallel to the Cartesian directions for the lower left quarter of the medium.

here. Efficient damping has been obtained with the values of $B=25$ and $C=2$ for equations (8). For an illustration of the PML mesh structure and of the numerical implementation of the wave-absorbing effects, a test was performed in a homogeneous infinite medium with a P-wave velocity of $2500 \mathrm{~m} / \mathrm{s}$, an S-wave velocity of $1558 \mathrm{~m} / \mathrm{s}$, and a density of $1500 \mathrm{~kg} / \mathrm{m}^{3}$ inside a finite grid. The simulation was performed at $4 \mathrm{~Hz}$ with a unitary explosive source. Figure 3 shows the frequency map solution for (a) a non-constrained mesh, and (b) a constrained mesh. Only the real part of the horizontal velocity is shown, although the behaviour is similar for the other components of the solutions. The mesh structure clearly has a significant influence on the PML efficiency.

\subsection{Source implementation}

The introduction of both point forces and point excitation stresses in the first-order system of equations (1) allows us to develop various point excitations: an impulsive force along the Cartesian directions or an explosive source can be applied easily using forces and stresses, respectively. A staggered behaviour over the mesh occurs in discrete equations, where the unknowns of one cell do not depend directly on the unknowns of its neighbours, but on the unknowns of the neighbours of the neighbouring cells. For particular mesh configurations, such as a regular equilateral mesh, if the source excitation is applied to one cell, simulations show that in the mesh only one cell out of two is excited. To avoiding this checker-board pattern, we spread the source over several cells using a Gaussian function. Of course, the numerical dispersion will express its properties based on twice the coarseness of the grid, as this is the price to be paid when considering centred fluxes. A similar observation can be made for the $O\left(\Delta x^{2}\right)$ rotated FD scheme. This has been the main reason for different authors moving to a staggered grid formulation. Figure 5 shows this pattern for an infinite homogeneous model with a central horizontal force. A single cell is excited in Figure 5-(a), whereas a Gaussian source is applied to 29 cells in Figure 5-(b). It can also be noted that for the model with a free surface, the explicit formulation of such a boundary recouples the two decoupled submeshes, as shown in Figure 6 with the same source configuration as in the previous test. We still need to implement smooth source excitation for propagation inside the medium.

\section{NUMERICAL RESULTS}

Several benchmarks will be presented to assess the accuracy of this new method, and we will focus more specifically on the influence of the mesh structure. Indeed, numerical tests with regular equilateral meshes and arbitrary unstructured meshes have shown the influence of the mesh regularity on solution accuracy: in spite of mesh refining, the solutions of the simulations do not converge to the true solution when considering unstructured meshes, whereas convergence is achieved with regular meshes. A sensitivity study on perturbed regular meshes has confirmed this behaviour. Accuracy problems in unstructured meshes typically involve travel-time shifts in seismograms. Dolean et al. 

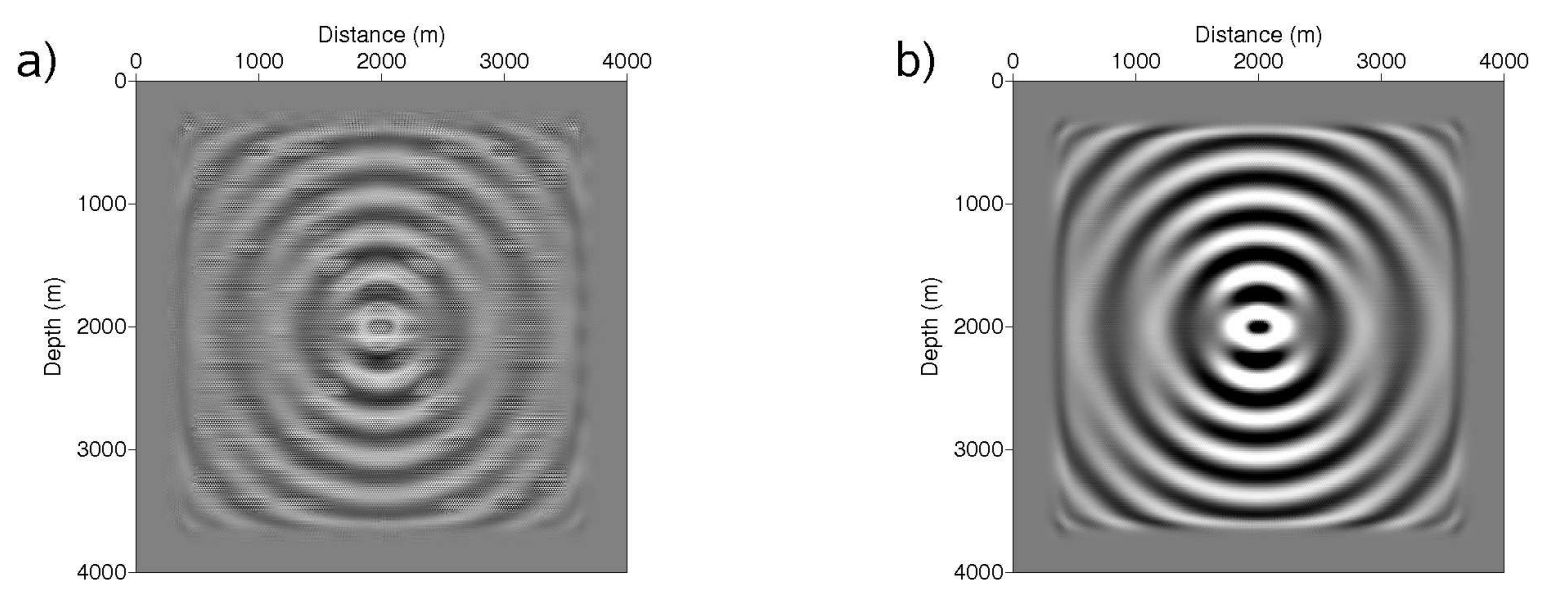

Figure 5. Frequency map solutions with the real parts of the horizontal velocity for a horizontal point force in an infinite medium. (a) Single cell excitation, and (b) smooth source excitation on several cells (29).

a)

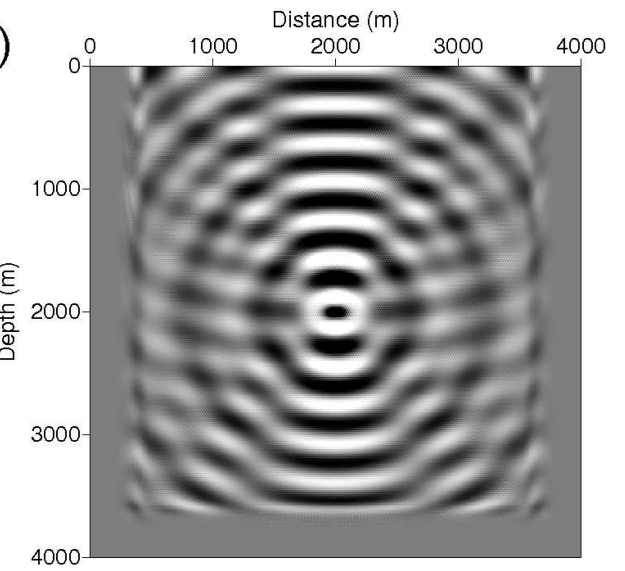

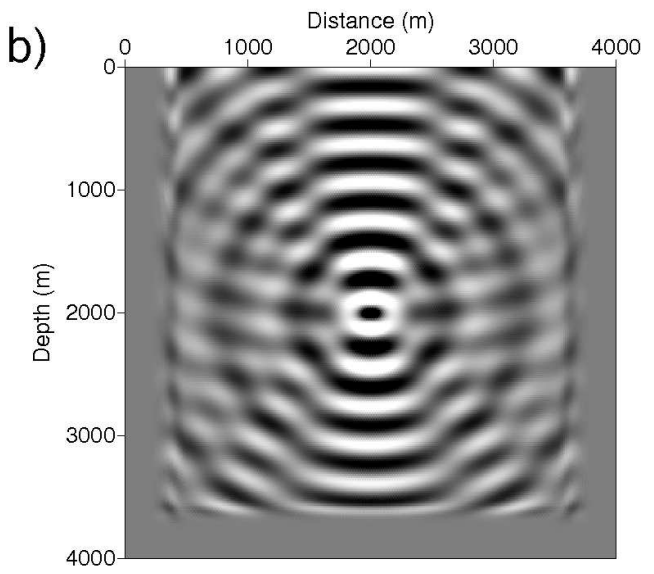

Figure 6. Frequency map solution with real parts of the horizontal velocity for a horizontal point force in a flat topography model. (a) Single cell excitation, and (b) smooth source excitation on several cells (29). The mesh pattern is less visible than for Figure 5, although it is still present in (a).

(2006) observed the same behaviour of convergence dependency for $P_{0}$ interpolation when applied to Maxwell equations in the frequency domain, while convergence of a linear $P_{1}$ interpolation appears to be less dependent on the regularity of the mesh. Moreover, the theoretical evaluation of the second-order accuracy in space of the numerical scheme has been demonstrated for regular structured meshes (Remaki, 2000). In our different comparisons, we will specify when we consider regular equilateral meshes or unstructured meshes. In the following, we first compare the FV results with the analytical solutions for different canonical problems. The solutions to more complex models are then compared with FD solutions.

Analytical solutions and reference FD codes have been constructed in the time domain, and comparisons are performed in this domain. FV solutions are, of course, computed for several frequencies, spanning over the source wavelet bandwidth. To avoid the wrap-around effect in seismograms, complex frequencies (Mallick and Frazer, 1987) are used in frequency-domain simulations. An inverse fast Fourier transformation will provide us with seismograms in the time domain for the comparison of the solutions. The size of the cells is chosen with respect to the maximal frequency of the source bandwidth. The results for the unstructured meshes are finally illustrated.

\subsection{Comparison with analytical solutions}

\subsubsection{An infinite homogeneous model with an explosive source: the acoustic case}

Acoustic propagation can be modelled inside an infinite homogeneous elastic medium using an explosive source that generates only a P-wave pulse. The numerical grid is bounded by four absorbing layers on the edges. For the homogeneous case, the analytical solutions are build up for the Helmholtz equation. A comparison of the radial velocity with the analytical solution was performed for a $2500 \mathrm{~m} / \mathrm{s}$ P-wave velocity medium with a regular equilateral mesh of size 1/10 of the P-wavelength (no S-wave is generated). Figure 7 shows the seismogram of the 


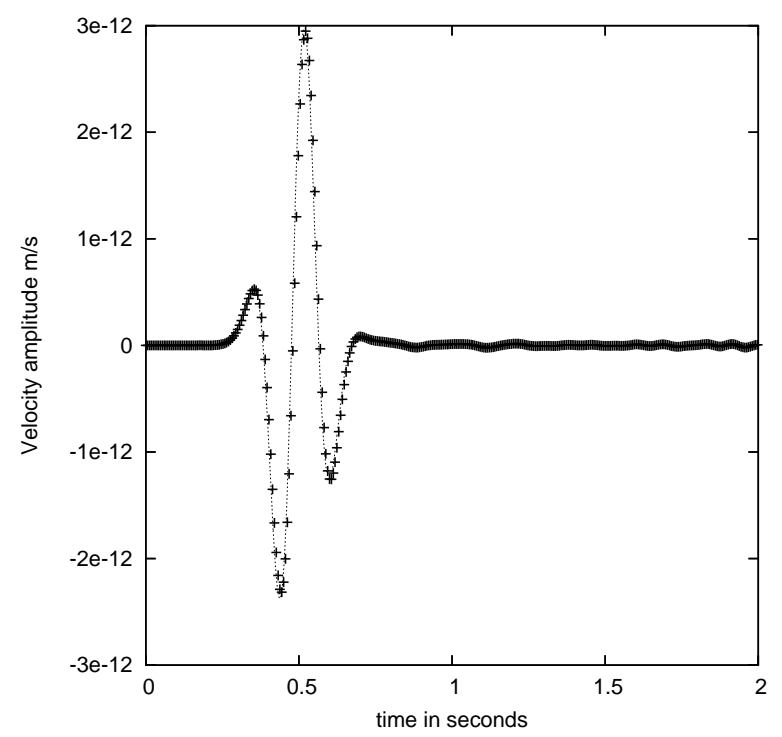

Figure 7. Comparison between analytical (continuous line) and numerical (crosses) seismograms for the acoustic case.

radial velocity at a receiver at a distance of $500 \mathrm{~m}$ from the source, and it illustrates the good agreement between the FV (crosses) and analytical (continuous line) seismograms. The tangential velocity is not strictly equal to zero, due to the smooth source and the numerical errors, although it still remains negligible.

\subsubsection{Flat free-surface medium: the Garvin problem}

The Garvin analytical solution deals with the propagation of elastic waves in a homogeneous half space with a flat free surface and an explosive source (Garvin, 1956). Comparisons with a Garvin solution are quite a challenging problem because the FV method must model Rayleigh waves with good accuracy near to the free surface, a critical issue for an efficient full waveform inversion algorithm. A homogeneous medium with a P-wave velocity of $3464 \mathrm{~m} / \mathrm{s}$, an S-wave velocity of $2000 \mathrm{~m} / \mathrm{s}$, and a density of $2000 \mathrm{~kg} / \mathrm{m}^{3}$ was considered. A Gaussian explosive source was considered at $150 \mathrm{~m}$ in depth, with $15 \mathrm{~m}$ of correlation length and with a line of receivers set on the surface with an offset of $200 \mathrm{~m}$ to $4000 \mathrm{~m}$, and a space step of $200 \mathrm{~m}$. A Ricker wavelet of central frequency $4 \mathrm{~Hz}$ was chosen. The simulation was performed over the source bandwidth, ranging from $0 \mathrm{~Hz}$ to $14 \mathrm{~Hz}$. A regular equilateral mesh was taken, with a cell edge size of $15 \mathrm{~m}$, which represents $1 / 10$ of the S-wavelength.

The horizontal and vertical particle-velocity seismograms computed with the FV method are compared with the analytical ones in Figure 8. The direct and the Rayleigh waves are both modelled with very good levels of accuracy in shape and amplitude for the whole range of offsets for the two components. No time shift appears with the offsets, which confirms the small dispersion of the scheme when considering 10 cells per S-wavelength. Since our seismograms have relative simple shapes, we have considered the relative RMS residuals of the particle velocity field in the time domain for a quantitative estimation of the accuracy of the numerical solutions. The relative residual at one observer is the L2 norm of one component of the differential seismograms over the L2 norm of the same component of the reference seismogram. Moreover, we consider the total relative residual, named the TRR value, as the average of the relative residuals over the receivers. The TRR values are $4.110^{-2}$ and $2.610^{-2}$ for the horizontal and vertical components, respectively.

\subsubsection{The two-layer model with a horizontal interface}

An analytical solution can be constructed when considering two homogeneous half spaces. A compressional point source will act in the upper layer. The software code known as EX2DELEL and provided by the Spice consortium (http://www.spice-rtn.org) was used to compute these solutions. Green's functions were first computed by the Cagniard-De Hoop technique, and a numerical convolution with the source wavelet gave the total response. The FV simulation was performed with the PML conditions on the four edges of the model for considering an infinite medium. The model dimensions were $12 \times 2.5 \mathrm{~km}$. The interface between the two layers was at a depth of $1150 \mathrm{~m}$. The receivers were placed on a line at a depth of $280 \mathrm{~m}$, with a space step of $200 \mathrm{~m}$, from $0 \mathrm{~m}$ to $12000 \mathrm{~m}$ in distance, leading to an array of 201 sensors. The explosive source was placed at a distance of $500 \mathrm{~m}$ and at a depth of $370 \mathrm{~m}$, with a correlation length of $30 \mathrm{~m}$. The source wavelet was a Ricker wavelet with a central frequency of $4 \mathrm{~Hz}$. Two tests were performed in regular equilateral meshes to evaluate the fluid-solid and solid-solid interfaces.

Fluid-solid interface 

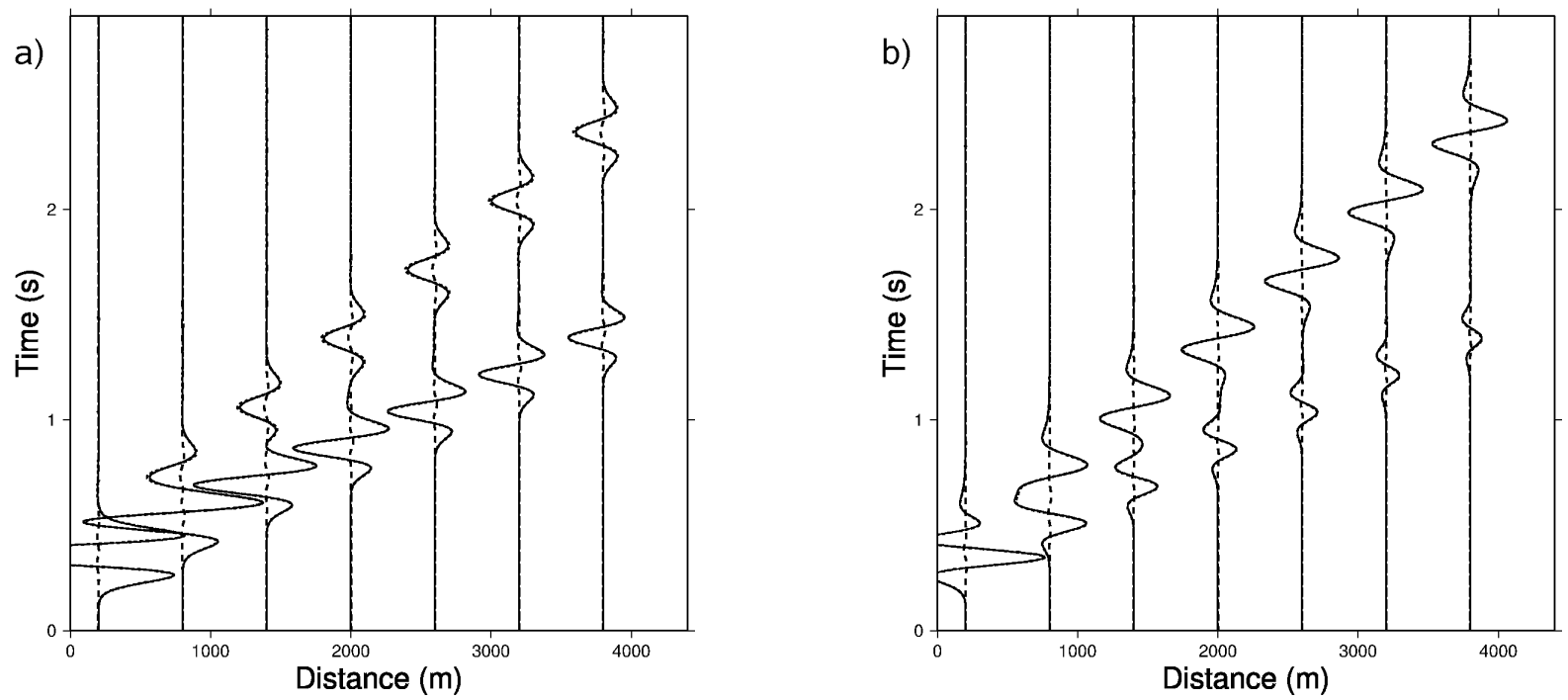

Figure 8. Seismograms for the Garvin problem. (a) Horizontal and (b) vertical components of the velocity at the receivers. The analytical solution is represented by dotted lines, FV by continuous lines, and the differences by dashed lines.
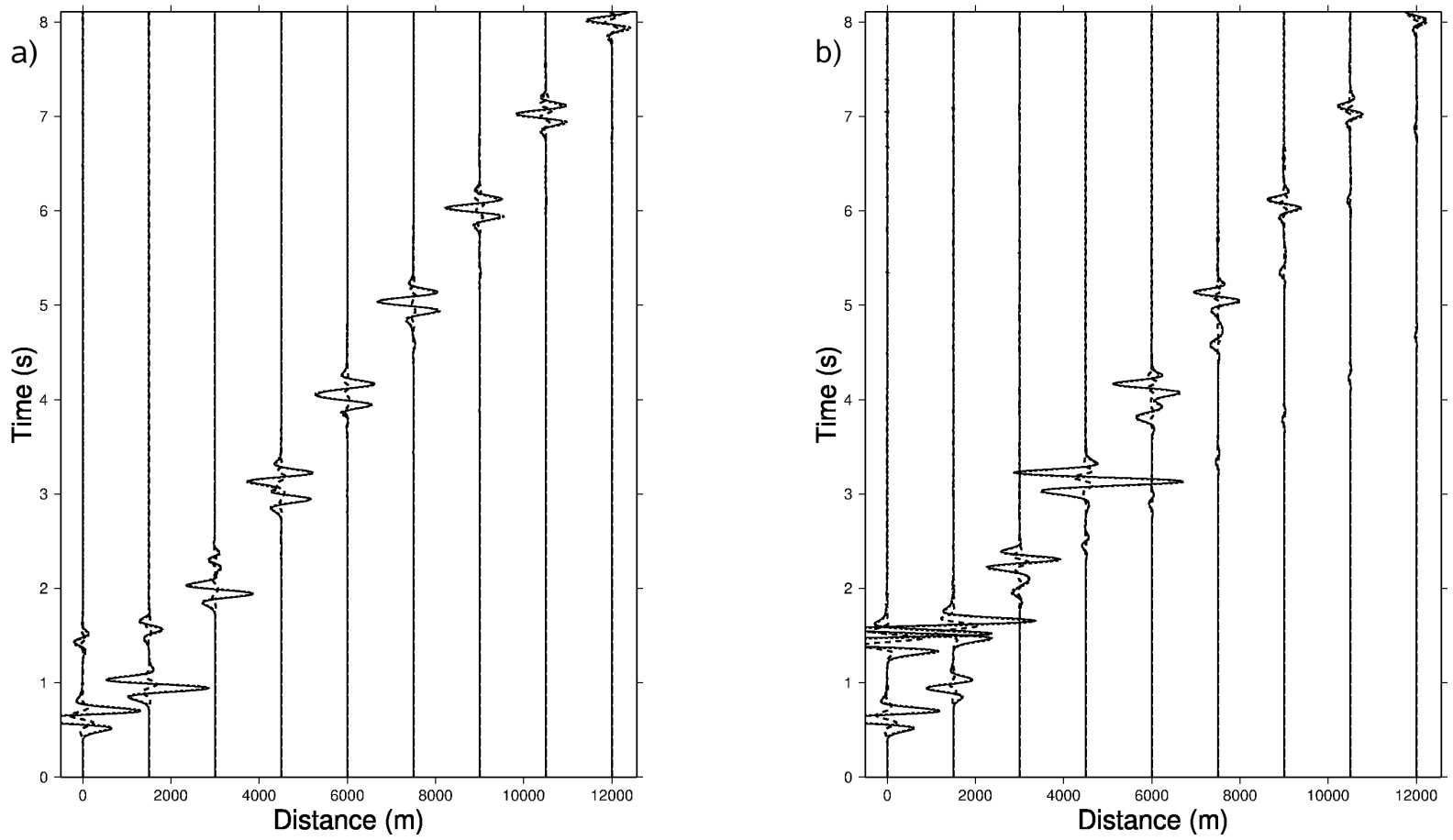

Figure 9. Seismograms computed in the two-layer model with a liquid-solid interface. (a) Horizontal and (b) vertical components of the velocity at the receivers. The analytical solution is represented by dotted lines, FV by continuous lines, and the differences by dashed lines.

Fluid-solid interface modelling is quite challenging for marine acquisition or simulation in reservoirs, as discontinuities must exist at the boundary. It is important to verify the accuracy of the solution, and we considered a simple planar interface. The upper medium was considered as an acoustic one with a P-wave velocity of $1500 \mathrm{~m} / \mathrm{s}$, an S-wave velocity of $0 \mathrm{~m} / \mathrm{s}$, and a density of $1,000 \mathrm{~kg} / \mathrm{m}^{3}$, while the lower medium had a P-wave velocity of $3400 \mathrm{~m} / \mathrm{s}$, an S-wave velocity of $1963 \mathrm{~m} / \mathrm{s}$, and a density of $2400 \mathrm{~kg} / \mathrm{m}^{3}$. A discretisation of 13 cells per P-wavelength in the fluid domain was chosen to keep the numerical dispersion negligible. Figure 9 shows the horizontal and vertical components of the particle velocity for the analytical (dotted), FV (continuous) and residual (dashed) solutions. There is good agreement between the analytical and FV solutions. The TRR values are $15.210^{-2}$ for the horizontal component, and $17.910^{-2}$ for the vertical one. However, we can see parasite reflections from PML zones exactly where the acoustic/elastic interface penetrates the PML layer. Figure 10 illustrates such reflections for a shorter offset model.

\section{Solid-solid interface}

The solid-solid interface test was performed with values of $2500 \mathrm{~m} / \mathrm{s}, 1558 \mathrm{~m} / \mathrm{s}$ and $1500 \mathrm{~kg} / \mathrm{m}^{3}$ for the P-wave velocity, the S-wave velocity, 

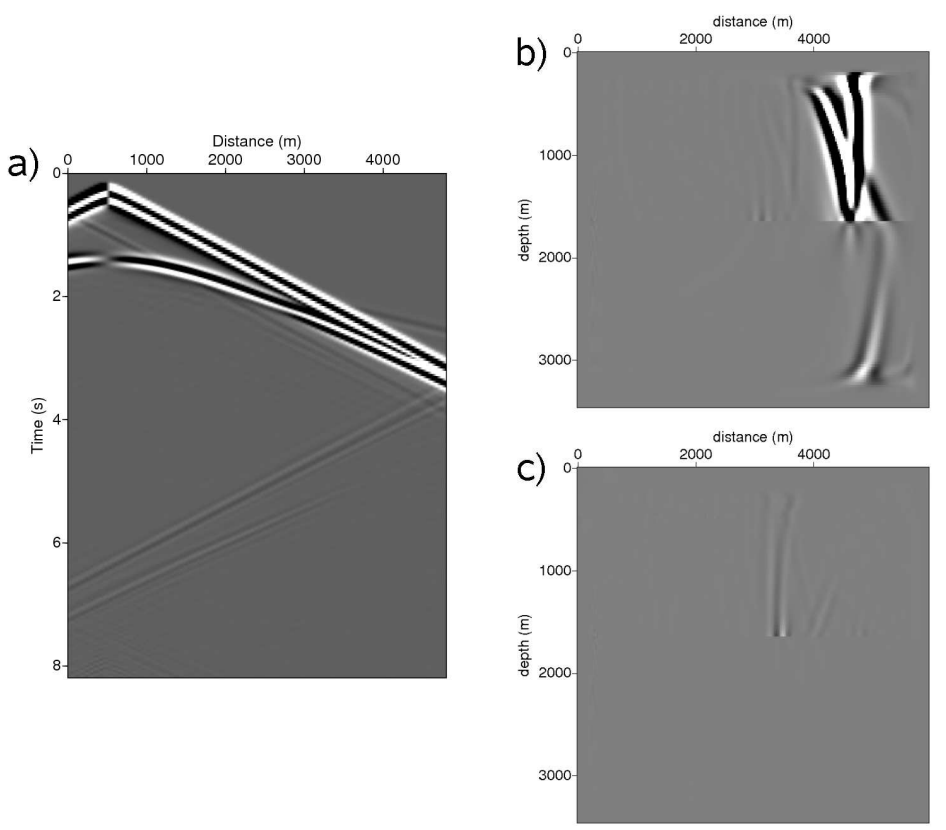

Figure 10. Seismograms at (a) receiver positions, and (b, c) snapshots for the horizontal component of the particle velocity for the two-layer problem with a liquid-solid interface and short offset geometry. Note the parasite reflections from the PML with a liquid-solid interface. The snapshots illustrate the incident wavefield at $2.8 \mathrm{~s} \mathrm{(b)} \mathrm{and} \mathrm{the} \mathrm{reflected} \mathrm{waves} \mathrm{from} \mathrm{the} \mathrm{PML} \mathrm{at} 4.8 \mathrm{~s}$ (c).
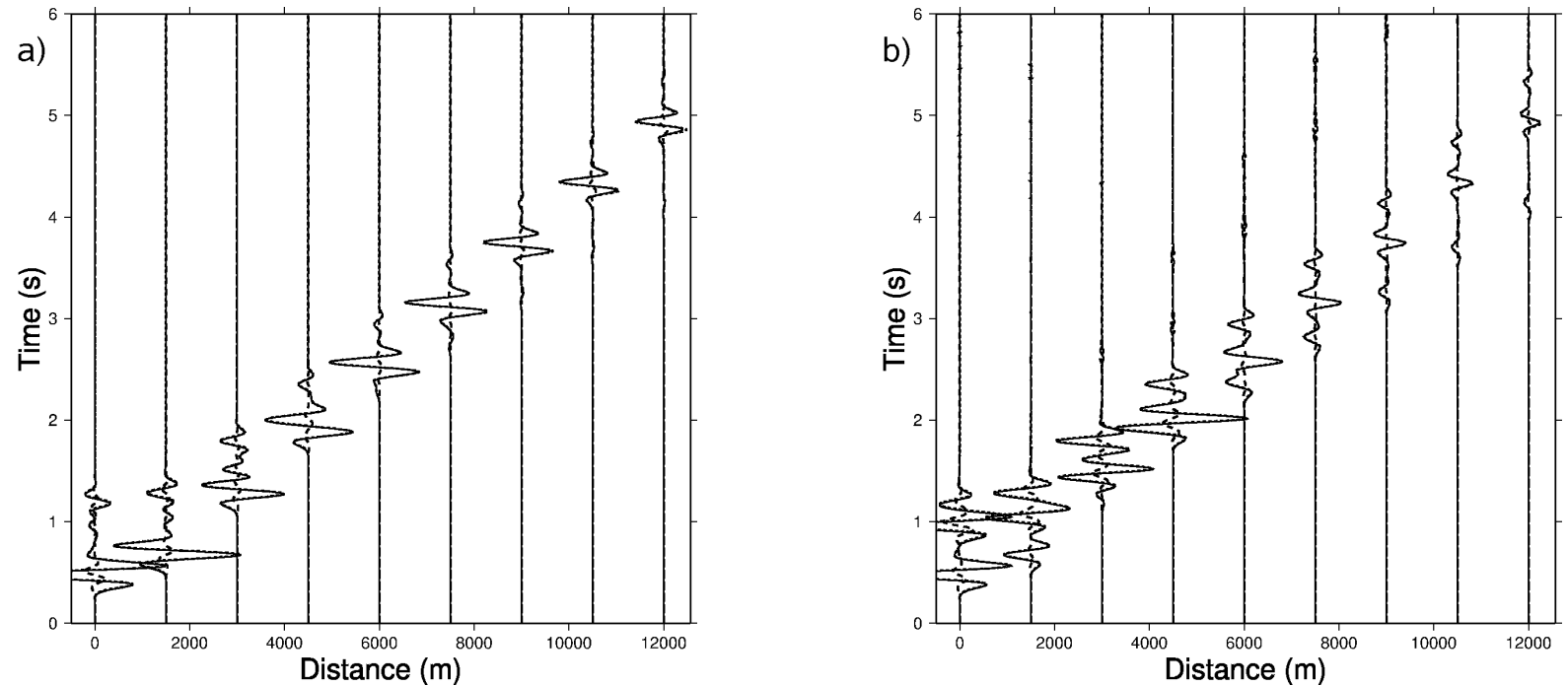

Figure 11. Seismograms computed for the two elastic-layer model. (a) Horizontal and (b) vertical components of the velocity at the receivers. The analytical solution is represented by dotted lines, FV by continuous lines, and the differences by dashed lines.

and the density for the upper half-space, and values of $3400 \mathrm{~m} / \mathrm{s}, 1963 \mathrm{~m} / \mathrm{s}$ and $2400 \mathrm{~kg} / \mathrm{m}^{3}$, respectively, for the lower half-space. The FV seismograms were computed in a regular mesh, with ten cells per shear wavelength. There is good agreement between the analytical and FV seismograms (Figure 11). The TRR values are $10.010^{-2}$ and $12.310^{-2}$ for the horizontal and vertical components, respectively. Note the efficient absorption of the PML in the case of the elastic-elastic interface.

\subsection{Comparison with numerical solutions}

The FV method needs to be benchmarked according to the other numerical techniques that can be applied to a more complex medium, which should be more representative of the realistic applications of full waveform inversion. The FV solutions were validated against seismograms computed with a time-domain $O\left(\Delta x^{2}, \Delta t^{2}\right)$ rotated staggered-grid FD method (Saenger et al., 2000), for three complex media: the corner- 


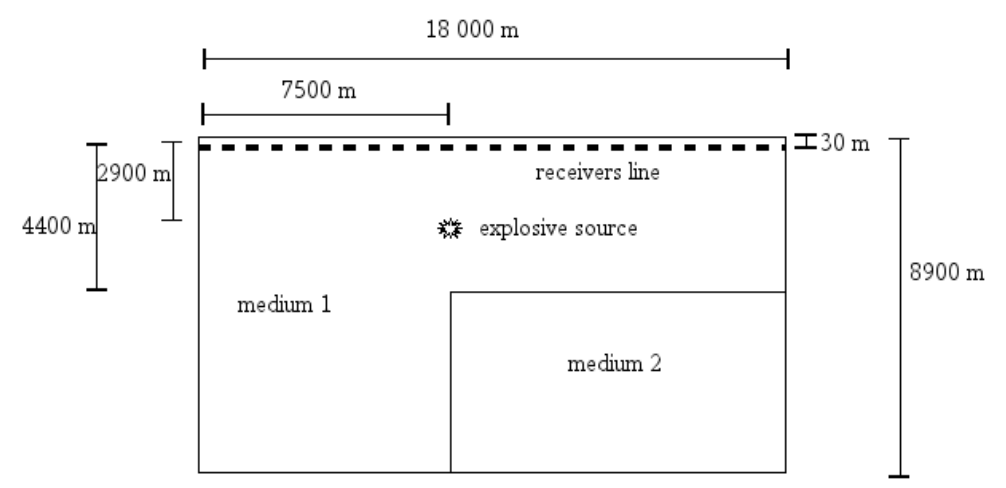

Figure 12. Geometry of the Corner-Edge model.
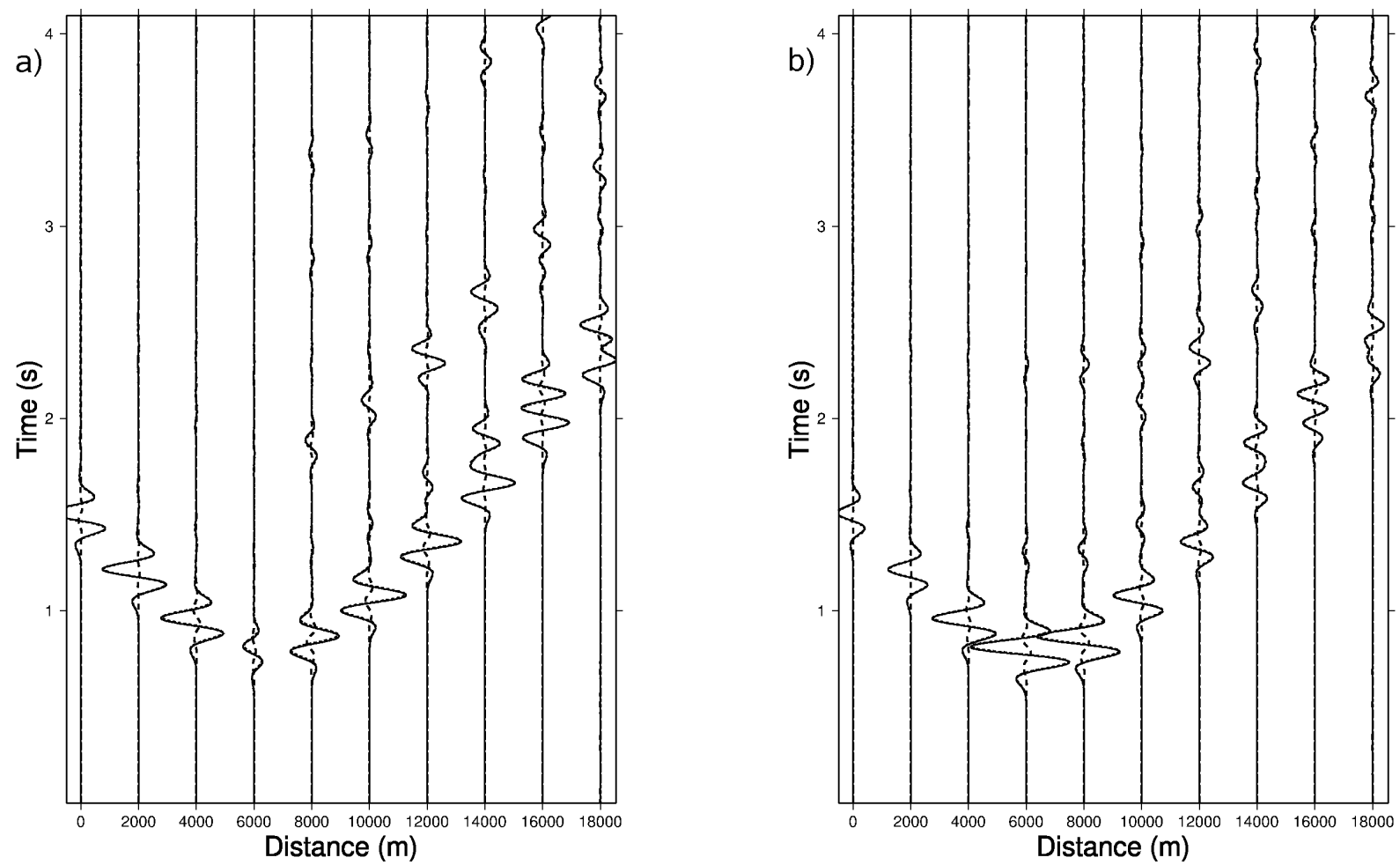

Figure 13. Seismograms computed for the Corner-Edge model. (a) Horizontal and (b) vertical components of the velocity at the receivers. The reference solution computed with the FD method is represented by dotted lines, FV by continuous lines, and the residuals by dashed lines. Both of these solutions are very similar across the entire time window.

edge model; a homogeneous hill model for considering complex topography; and a realistic heterogeneous model corresponding to a subset of the so-called Marmousi II model.

\subsubsection{The Corner-Edge Model}

A synthetic model, known as the corner-edge model (Figure 12), is defined by a flat free surface and a corner with a sharp velocity contrast that introduces multiple reflections and diffractions for both body and surface waves (Virieux, 1986a). The upper medium had a P-wave velocity of $6000 \mathrm{~m} / \mathrm{s}$, whereas the lower medium had a P-wave velocity of $9000 \mathrm{~m} / \mathrm{s}$. The S-wave velocity was computed from the P-wave velocity, with a ratio of $\sqrt{3}$. The model had a homogeneous density of $2500 \mathrm{~kg} / \mathrm{m}^{3}$. The explosive source was located at $(x=7500 \mathrm{~m}, \mathrm{z}=2900$ $\mathrm{m}$ ), and it had a Ricker wavelet of central frequency of $4 \mathrm{~Hz}$ as the time function. The receiver line was placed below the topography at a depth of $30 \mathrm{~m}$, with a receiver spacing of $50 \mathrm{~m}$, from $0 \mathrm{~m}$ to $18000 \mathrm{~m}$. An equilateral mesh was constructed with an edge length of 26.6 $\mathrm{m}$, corresponding to the discretisation rule of ten cells per minimum shear wavelength. The agreement between the FV and FD solutions is illustrated in Figure 13. The TRR values are $10.110^{-2}$ for the horizontal and $9.810^{-2}$ for the vertical components of the velocity. 


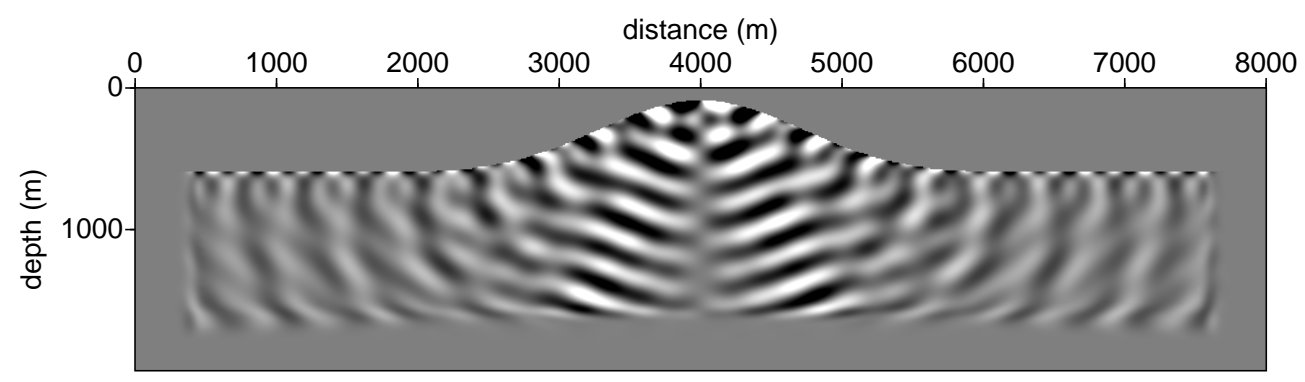

Figure 14. Monochromatic wavefield of the horizontal velocity for the hill model. The real part of the wavefield is illustrated for a 10- $\mathrm{Hz}$ simulation.

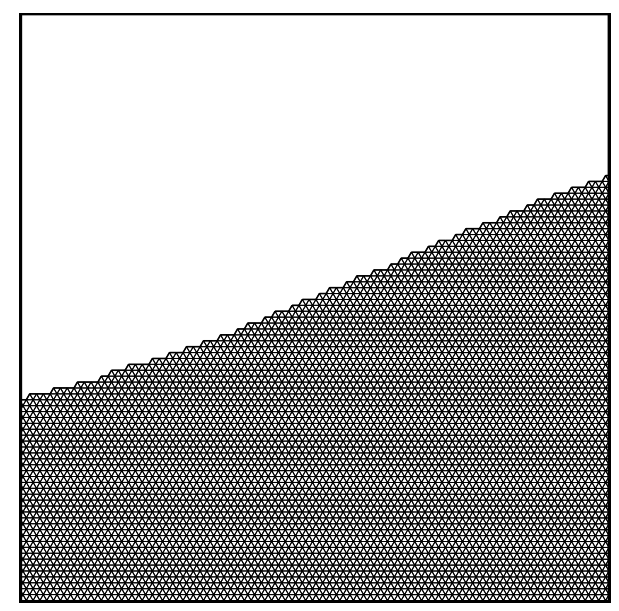

Figure 15. Description of a complex topography with regular equilateral triangles.

\subsubsection{Complex topography Model}

A homogeneous model with a hill-shaped topography was used to assess the accuracy of the FV method with a non-flat free surface. A homogeneous medium was used $\left(4000 \mathrm{~m} / \mathrm{s}\right.$ and $2309 \mathrm{~m} / \mathrm{s}$, for P-wave and S-wave velocities, respectively, and 2,000 kg/m $\mathrm{m}^{3}$ for density). An explosive source was set in the middle of the hill, $25 \mathrm{~m}$ below the topography, and it had a Ricker wavelet of central frequency of 4 $\mathrm{Hz}$ as a time dependence. The receiver line was located at a depth of $5 \mathrm{~m}$ below the free surface. Figure 14 shows the real part of a 10-Hz monochromatic wavefield for the horizontal velocity component. An equilateral mesh allows the modelling of the topography by straight lines (Figure 15), without the stair-case description of the FD methods. This description is not perfect, as it should be with an unstructured mesh, but numerical simulations have shown quite accurate results with 15 cells per shear wavelength with this topography, whereas the second-order rotated staggered-grid FD stencil requires 60 points. The FV and FD seismograms computed with these two above-mentioned discretisation rules (15 and 60 cells per shear-wavelength, respectively) are compared in Figure 16, and they show good agreement. The TRR values are $11.710^{-2}$ and $12.410^{-2}$ for the horizontal and vertical components of the velocity, respectively. The surface waves are well modelled, and no numerical dispersion occurs. Simulations with a finer mesh led to comparable seismograms for both the FV and the FD methods.

\subsubsection{Realistic model: a subset of the Marmousi II model}

The Marmousi II synthetic model represents a complex elastic medium, which makes it suitable for testing the FV method we propose here. A limited target of the model with multiple interfaces was chosen to limit the core memory requested by the frequency-domain formulation used for building the time-domain seismograms. This target, the dimensions of which were $5000 \mathrm{~m} \times 2000 \mathrm{~m}(6000 \mathrm{~m} \times 2500 \mathrm{~m}$ with the PML layers), is illustrated in Figure 17 for the P-wave velocities. An explosive source was located at ( $x=1000 \mathrm{~m}, \mathrm{z}=100 \mathrm{~m})$, and a Ricker wavelet of central frequency of $4 \mathrm{~Hz}$ was considered. The receiver line was set at a depth of $25 \mathrm{~m}$ below the topography. The edges of the regular triangular mesh had lengths of $7.1 \mathrm{~m}$, corresponding to 10 cells per shear wavelength. Figure 18 illustrates the seismograms at the receivers. Comparisons with the FD method show quite similar results. The small differences that occur for the horizontal velocity can be 

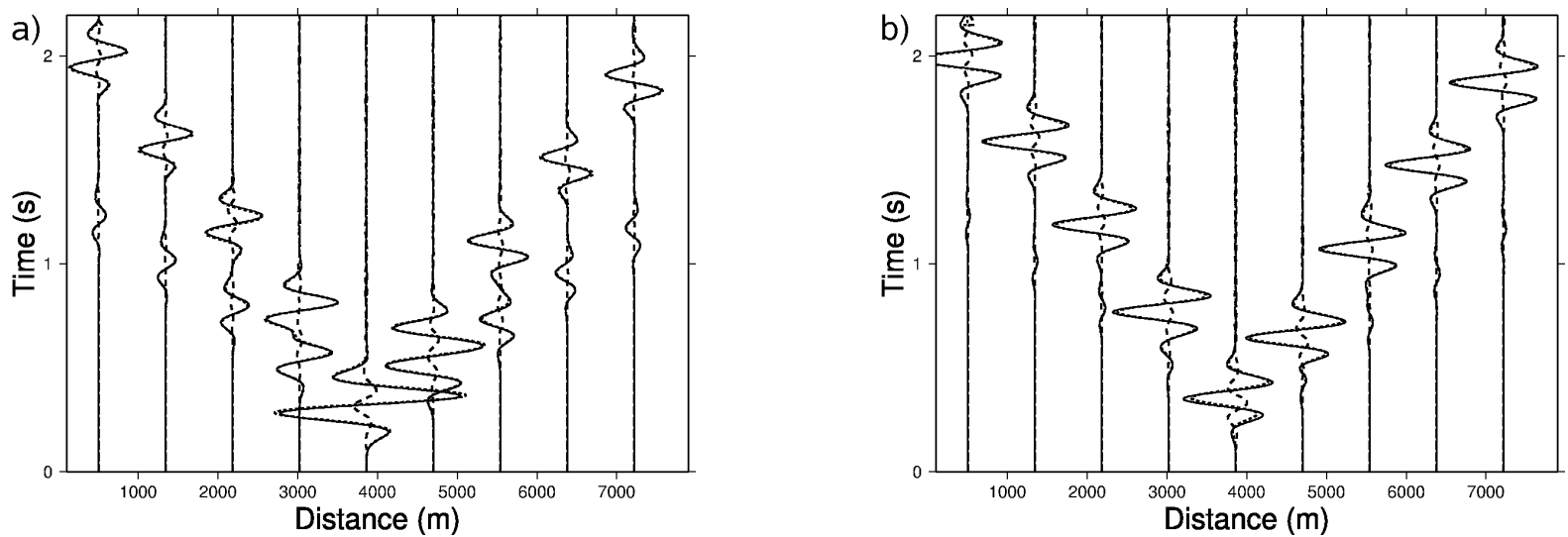

Figure 16. Seismograms computed for the hill model. (a) Horizontal and (b) vertical components of the velocity. The reference solution computed with the FD method is represented by dotted lines, the FV solution by continuous lines, and the difference between the two solutions with dashed lines.

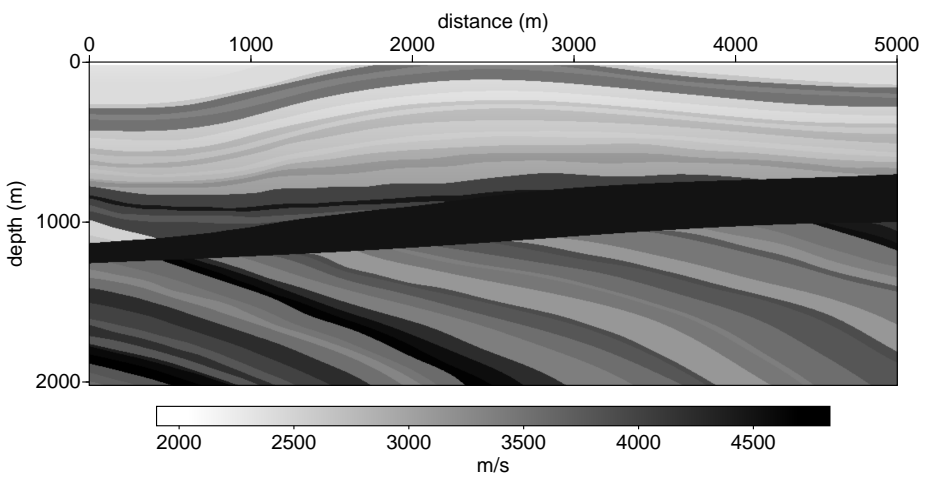

Figure 17. P-wave velocity distribution of the realistic model taken from the Marmousi II model.

attributed to the model description, which is slightly different for the square and the triangle parametrisation. The FV simulations in finer triangular meshes led to similar seismograms, hence providing additional validation of the discretisation rule of ten cells per S-wavelength for heterogeneous media, which is quite encouraging for future work.

\subsection{Numerical tests with unstructured meshes}

This FV method is now analysed in unstructured meshes. The $P_{0}$ interpolation should provide solutions with a given level of accuracy in such meshes. Previous hill models and Marmousi II models will be considered. The FV solutions computed for an equilateral mesh will be used as the reference solutions. Unstructured meshes allow very precise modelling of the free surface when a complex topography is considered. No constraints on triangle angles have been applied for either model. Moreover, the triangle sizes can be adapted to the propagated wavelengths locally, to minimise the number of cells, and therefore the number of unknowns to be solved in the linear system, a very appealing feature when performing factorisation of the impedance matrix. The hill model simulation was performed with an unstructured mesh by considering the discretisation rule of fifteen cells per minimum shear wavelength. The comparison between seismograms computed for an equilateral structured and for unstructured meshes is shown in Figure 19. Of course, there is a good match of the amplitudes, whereas there is a negative time shift of the phases that increases with the propagation time in seismograms computed in the unstructured mesh. The Marmousi II model simulation was performed in an unstructured mesh adapted to the local shear-wave velocity, with at least ten cells per shear wavelength. The seismograms look similar to those computed for an equilateral mesh (compare Figures 18 and 20). However, a direct comparison between the seismograms computed in the equilateral and unstructured meshes ( Figure 21) shows the same slight negative time delay as is seen for the hill model. The simulations in finer unstructured meshes for both the hill and Marmousi II models have not shown better convergence of the numerical solution, as expected for the $P_{0}$ interpolation for unstructured meshes.

\section{NUMERICAL COST OF METHOD}

Computational costs are always a difficult question, and we will focus here on a comparison between the FV and FD methods for the same order of accuracy in the frequency domain. We will focus on the specific example of the Marmousi II model (Figure 17). The source was 

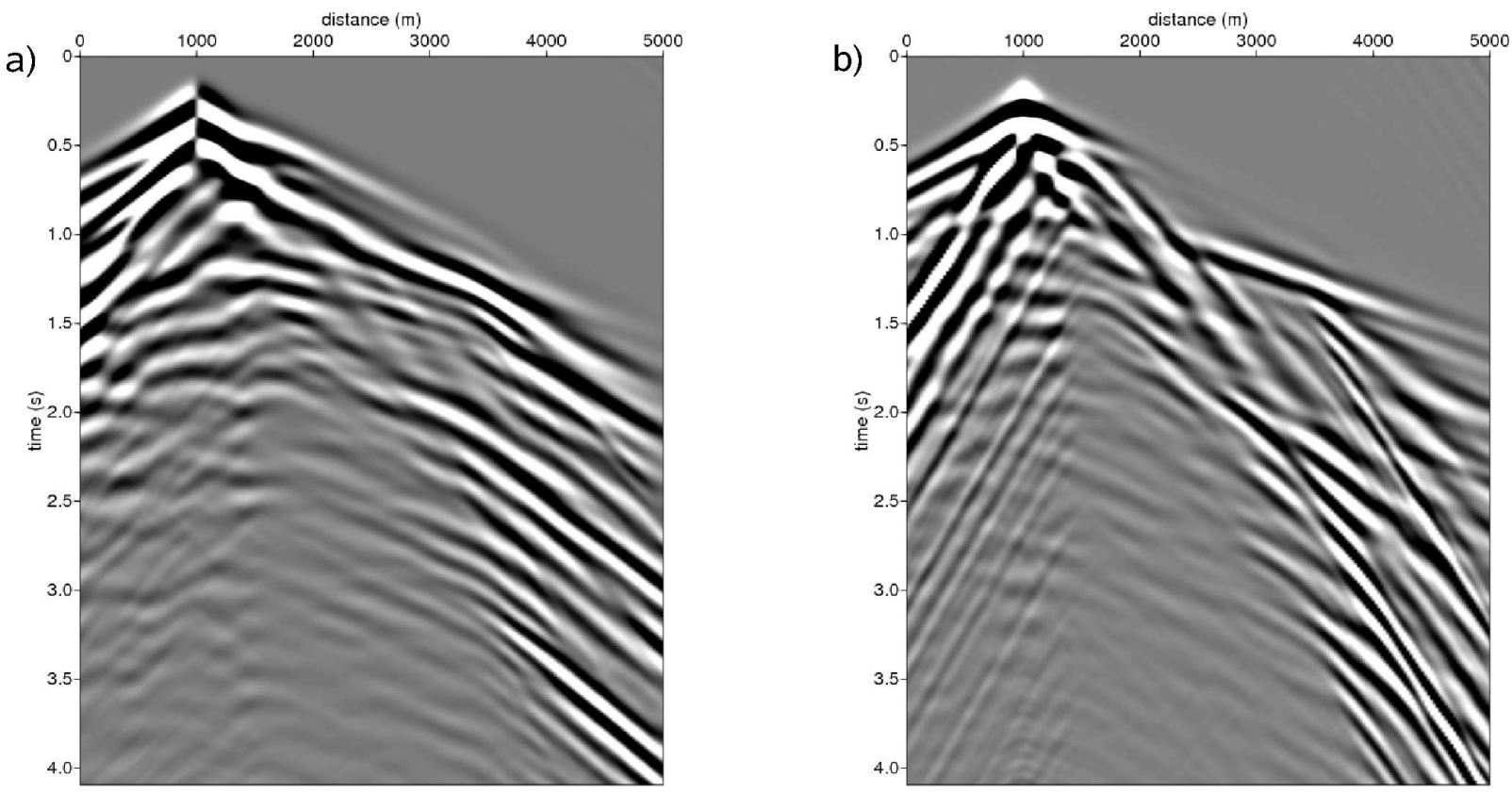

Figure 18. Seismograms computed in the target of the Marmousi II model with the FV method, using a regular equilateral mesh. (a) Horizontal and (b) vertical particle velocities at the receiver positions.
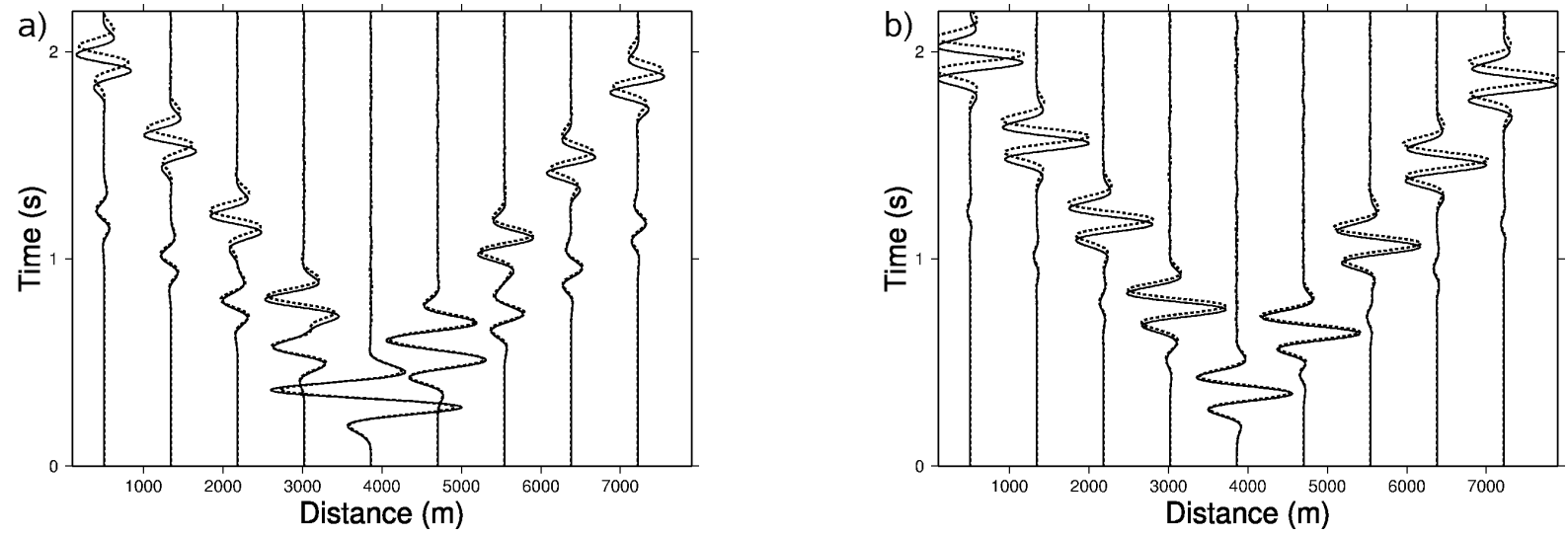

Figure 19. Seismograms computed in the hill model using equilateral and unstructured meshes. (a) Horizontal and (b) vertical components of the particle velocity at the receivers. The reference solution computed with FV in regular equilateral mesh is plotted with dotted lines, and the solution in an unstructured mesh with continuous lines. Note the advance of the solution computed in the unstructured mesh, as it increases with propagation time.

an explosion. The modelled frequency was $13 \mathrm{~Hz}$. We can illustrate the CPU time and memory requirements of the FV method with the second-order parsimonious rotated frequency-domain FD method (Gelis et al., 2007). Both of these methods make use of the direct solver MUMPS (MUMPS-team, 2007), which performs the resolution of the linear system by LU decomposition of the sparse matrix through a multifrontal approach. The medium is discretised with 10 cells per minimum S-wavelength for the FV approach in a regular equilateral mesh, with 10 cells per local S-wavelength for the FV method in an unstructured mesh, and with 28 points for the FD method in order to have an acceptable numerical dispersion.

Table 1 illustrates the requirements of both of these methods for sequential execution on a single processor. The coarser parameterisation of the FV naturally leads to less unknowns to be computed and a less expensive estimation in terms of CPU time and core memory for all of the MUMPS phases: a factor of 2.5 can be noted in this example, between the FV method in a regular mesh and the FD method. Moreover, an adaptive unstructured mesh allows for a significant decrease in the numerical resources when a heterogeneous medium is considered, although we must be aware of the approximate precision of the solution when performing the seismic inversion procedure. However, the mesh description of the medium introduces several additional table build-ups and manipulations for the matrix construction, which are more time consuming than the simple implicit construction of the FD techniques on a regular grid. Extra CPU time costs of the FV methods 

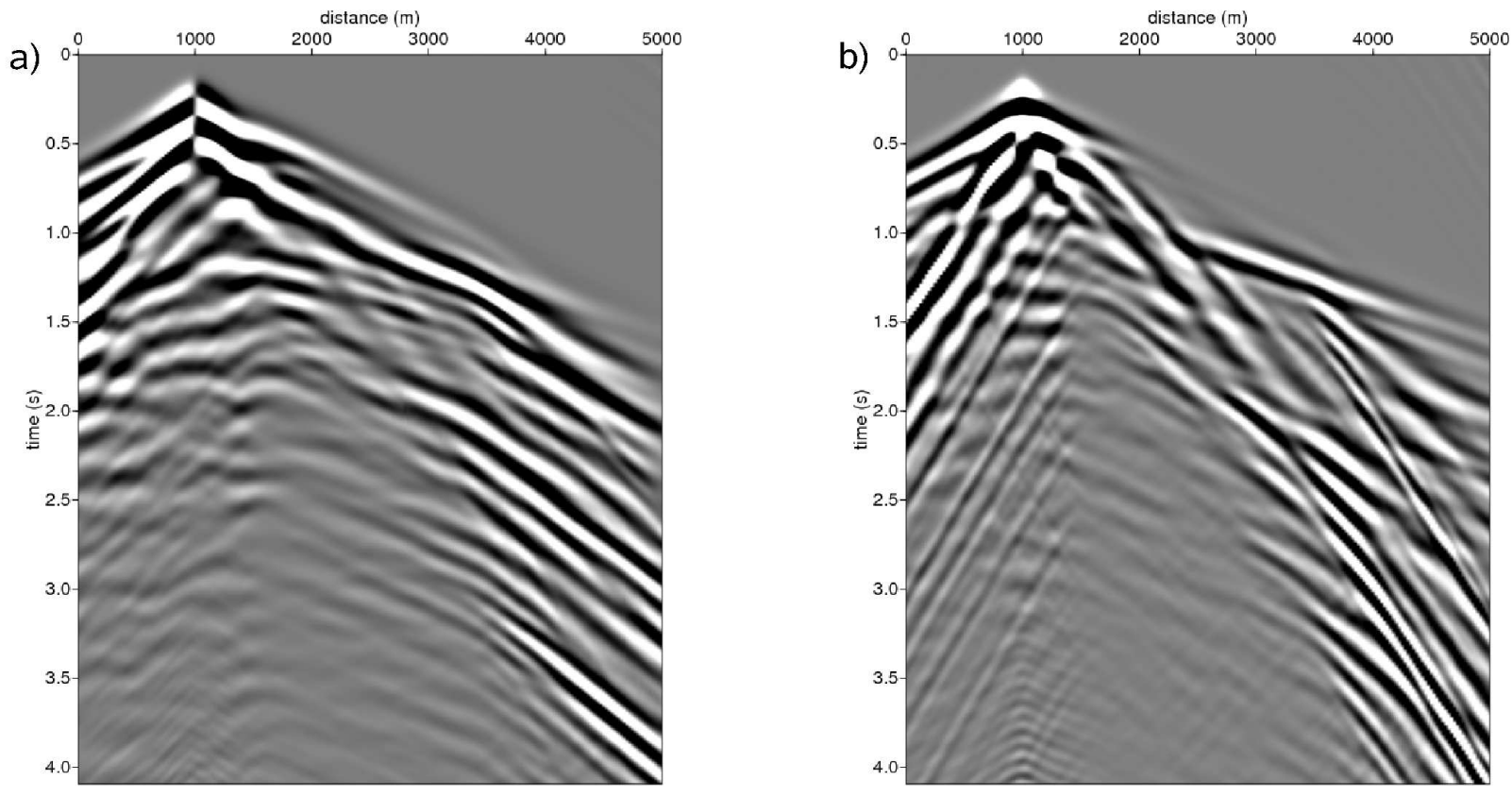

Figure 20. Seismograms computed in the target of the Marmousi II model using an unstructured mesh. (a) Horizontal and (b) vertical components of the velocity at the receivers. These seismograms can be compared with that computed in the same model parametrised with an equilateral mesh (Figure 18).
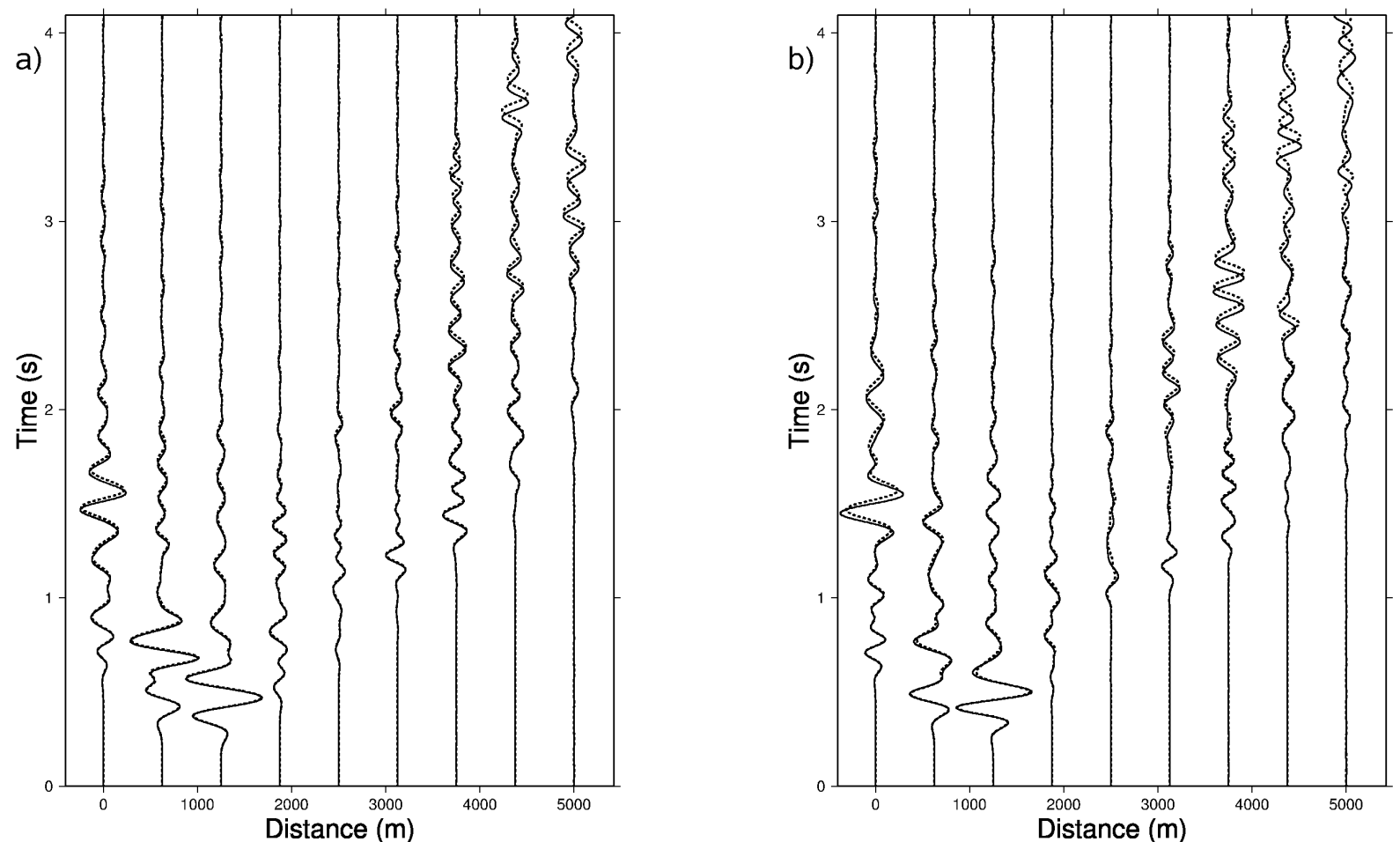

Figure 21. Comparison between the FV seismograms computed in the target of the Marmousi II model with equilateral and unstructured meshes. (a) Horizontal and (b) vertical components of the velocity at the receivers. The reference solution computed with FV in a regular equilateral mesh is represented by dotted lines, and the solution in an unstructured mesh by continuous lines. 
Table 1. Overview of the numerical cost for the FV method in regular and unstructured meshes and the $O\left(\Delta x^{2}\right)$ FD method in the frequency domain for a realistic model at $13 \mathrm{~Hz}$.

\begin{tabular}{cccc}
\hline Numerical method & regular FV & unstructured FV & FD \\
\hline \hline Number of unknowns to solve & 1421364 & 549638 & 4850020 \\
\hline Time to prepare data for matrix building (s) & 54.4 & 40.1 & 1.0 \\
\hline Time for matrix building (s) & 1.6 & 0.80 & 10.9 \\
\hline Time for factorisation (s) & 272.3 & 79.5 & 999.4 \\
\hline Memory use for factorisation (Mb) & 3448 & 1333 & 12061 \\
\hline Time for resolution of 1 shot (s) & 3.8 & 1.7 & 13.5 \\
\hline
\end{tabular}

will remain small for the different MUMPS numerical procedures, and will occur only once when considering a full waveform inversion algorithm; they will not hamper the benefit of using a coarser grid for the FV method.

\section{DISCUSSION AND CONCLUSION}

A FV method dedicated to full waveform inversion has been formulated in the space-frequency domain for 2D P-SV wave propagation. By using the parsimonious approach, only particle velocity unknowns are used in the build-up of the impedance matrix, which incorporates various medium properties, including a free surface and possible liquid-solid interfaces. Comparisons between numerical solutions computed with analytic and numerical reference solutions for canonical and realistic configurations have shown that a structured equilateral mesh provides accurate results for a discretisation of 10 cells per shear wavelength, even if the topography and surface waves are considered. A complex topography should require a finer description of 15 cells, coarser than $O\left(\Delta x^{2}\right)$ FD due to the triangular meshing. Unstructured meshes are easily taken into account in the $P_{0} \mathrm{FV}$ formulation, although they suffer from a lack of precision even when fine meshes are considered. The CPU/memory requirements are naturally less expensive than for $O\left(\Delta x^{2}\right) \mathrm{FD}$, in spite of the complex table manipulations due to the mesh description of medium. Finally, the FV method with regular meshes appears to be very efficient when compared with $O\left(\Delta x^{2}\right)$ FD methods, especially when a realistic topography is considered. Considering unstructured meshes allows a significant decrease in the numerical resources; however, with lower accuracy of the wavefield approximation. Future work will focus on the accuracy needed in forward modelling for the application of full waveform inversion. We will investigate further the usefulness of unstructured meshes and the correlated accuracy for such an imaging strategy. As an alternative, moving to higher order for the interpolation in the discontinuous Galerkin approach will be a possibility. By considering the $P_{1}$ interpolation, we may find the best compromise between accuracy for the wavefield estimation and computational efficiency required by the inversion.

\section{ACKNOWLEDGMENTS}

This study was funded by the SEISCOPE consortium http://seiscope.unice.fr, which is sponsored by BP, CGG-VERITAS, EXXON-MOBIL, SHELL and TOTAL, and by Agence Nationale de la Recherche (ANR) under project ANR-05-NT05-2_42427. The LU factorisation of the impedance matrix was performed with the MUMPS package, available on http://graal.ens-lyon.fr/MUMPS/index.html. The mesh generation was performed with the help of TRIANGLE, available on http://www.cs.cmu.edu/ quake/triangle.html. The Garvin problem analytical solutions were computed with code provided by U. Iturrarán and F. J. Sánchez-Sesma. The two-layer analytic solutions were computed with the EX2DELEL code, available on http://www.spice-rtn.org/library/software/EX2DELEL. Many thanks go to Mondher Ben Jemaa, Nathalie Glinsky, Stéphane Lantéri and Serge Piperno of the CAIMAN project for interesting and stimulating discussions on the DG Pk approach. Access to the high-performance computing facilities of the MESOCENTRE SIGAMM computer centre provided the required computer resources, and we gratefully acknowledge both this facility and the support of the staff, and in particular of A. Miniussi. Reviews by P. Moczo and J. Robertsson significantly improved the first version of this manuscript.

\section{References}

BenJemaa, M., Glinsky-Olivier, N., Cruz-Atienza, V. M., Virieux, J., and Piperno, S. (2007). Dynamic non-planar crack rupture by a finite volume method. Geophysical Journal International, 171:271-285.

Berenger, J.-P. (1994). A perfectly matched layer for absorption of electromagnetic waves. Journal of Computational Physics, 114:185-200. Bielak, J., Loukakis, K., Hisada, Y., and Yoshimura, C. (2003). Domain Reduction Method for Three-Dimensional Earthquake Modeling in Localized Regions, Part I: Theory. Bulletin of the Seismological Society of America, 93(2):817-824. 
Bohlen, T. and Saenger, E. H. (2006). Accuracy of heterogeneous staggered-grid finite-difference modeling of Rayleigh waves. Geophysics, 71:109-115.

Casadei, F., Gabellini, E., Fotia, G., Maggio, F., and Quarteroni, A. (2002). A mortar spectral/finite element method for complex 2D and 3D elastodynamic problems. Comput. Method Appl. Mech. Engrg, 191:5119-5148.

Chaljub, E., Capdeville, Y., and Vilotte, J. (2003). Solving elastodynamics in a fluid-solid heterogeneous sphere: a parallel spectral element approximation on non-conforming grids. J. Compu. Phys., 187:457-491.

Chew, W. C. and Liu, Q. H. (1996). Perfectly matched layers for elastodynamics: a new absorbing boundary condition. J. Compu. Acous., 4:341-359.

Cockburn, B., Karniadakis, G., and Shu, C. W. (2000). Discontinuous Galerkin Method: Theory, Computation and Application. Springer - Lecture Notes in Computational Science Engineering.

de la Puente, J., Käser, M., Dumbser, M., and Igel, H. (2007). An Arbitrary High Order Discontinuous Galerkin Method for Elastic Waves on Unstructured Meshes IV: Anisotropy. Geophysical Journal International, 169(3):1210-1228.

Dolean, V., Fol, H., Lanteri, S., and Piperno, S. (2006). Méthode de type galerkin discontinu pour la résolution numérique des équations de Maxwell en régime fréquentiel. Rapport de Recherche 5904, INRIA Sophia-Antipolis.

Drossaert, F. H. and Giannopoulos, A. (2007). A nonsplit complex frequency-shifted PML based on recursive integration for FDTD modeling of elastic waves. Geophysics, 72(2):T9-T17.

Dumbser, M. and Käser, M. (2006). An Arbitrary High Order Discontinuous Galerkin Method for Elastic Waves on Unstructured Meshes II: The Three-Dimensional Isotropic Case. Geophysical Journal International, 167(1):319-336.

Dumbser, M., Käser, M., and Toro, E. (2007). An Arbitrary High Order Discontinuous Galerkin Method for Elastic Waves on Unstructured Meshes V: Local Time Stepping and p-Adaptivity. Geophysical Journal International, 171(2):695-717.

Faccioli, E. F., Paolucci, R., and Quarteroni, A. (1997). 2D and 3D elastic wave propagation by a pseudo-spectral domain decomposition method. J. Seismol., 1:237-251.

Garvin, W. W. (1956). Exact transient solution of the buried line source problem. Proc. Roy. Soc. London, 234:528-541.

Gauthier, O., Virieux, J., and Tarantola, A. (1986). Two-dimensional nonlinear inversion of seismic waveforms: numerical results. Geophysics, 51(7):1387-1403.

Gelis, C., Virieux, J., and Grandjean, G. (2007). 2D elastic waveform inversion using Born and Rytov approximations in the frequency domain. Geophysical Journal International, 168:605-633.

Hestholm, S. and Ruud, B. (2002). 3D free-boundary conditions for coordinate-transform finite-difference seismic modelling. Geophysical Prospecting, 50:463-474.

Hustedt, B., Operto, S., and Virieux, J. (2004). Mixed-grid and staggered-grid finite difference methods for frequency domain acoustic wave modelling. Geophysical Journal International, 157:1269-1296.

Jo, C. H., Shin, C., and Suh, J. H. (1996). An optimal 9-point, finite-difference, frequency-space 2D scalar extrapolator. Geophysics, 61:529-537.

Käser, M. and Dumbser, M. (2006). An Arbitrary High Order Discontinuous Galerkin Method for Elastic Waves on Unstructured Meshes I: The Two-Dimensional Isotropic Case with External Source Terms. Geophysical Journal International, 166:855-877.

Käser, M., Dumbser, M., de la Puente, J., and Igel, H. (2007). An Arbitrary High Order Discontinuous Galerkin Method for Elastic Waves on Unstructured Meshes III: Viscoelastic Attenuation. Geophysical Journal International, 168(1):224-242.

Koketsu, K., Fujiwara, H., and Ikegami, Y. (2004). Finite-element Simulation of Seismic Ground Motion with a Voxel Mesh. Pure and Applied Geophysics, 161:2183-2198.

Komatitsch, D. and Vilotte, J. P. (1998). The spectral element method: an efficient tool to simulate the seismic response of 2D and 3D geological structures. Bull. Seismol. Soc. Am., 88:368-392.

Levander, A. R. (1988). Fourth-order finite-difference P-SV seismograms. Geophysics, 53(11):1425-1436.

LeVeque, R. J. (2007). Finite Difference Methods for Ordinary and Partial Differential Equations, Steady State and Time Dependent Problems. SIAM.

Lombard, B., Piraux, J., Gelis, C., and Virieux, J. (2008). Free and smooth boundaries in 2-D finite-difference schemes for transient elastic waves. Geophysical Journal International, 172:252-261.

Luo, Y. and Schuster, G. T. (1990). Parsimonious staggered grid finite-differencing of the wave equation. Geophysical Research Letters, 17(2):155-158.

Madariaga, R. (1976). Dynamics of an expanding circular fault. Bull. Seismol. Soc. Am., 66:639-666.

Mallick, S. and Frazer, L. N. (1987). Practical aspects of reflectivity modeling. Geophysics, 52:1355-1364.

Marfurt, K. (1984). Accuracy of finite-difference and finite-elements modeling of the scalar and elastic wave equation. Geophysics, 49:533-549.

Moczo, P., Bystricky, E., Carcione, J. M., and Bouchon, M. (1997). Hybrid modeling of P-SV seismic motion at inhomogeneous viscoelastic topographic structures. Bull. Seismol. Soc. Am., 87:1305-1323.

MUMPS-team (2007). MUMPS - MUltifrontal Massively Parallel Solver users' guide - version 4.7 (April 2007). ENSEEIHT-ENS Lyon, http://www.enseeiht.fr/apo/MUMPS/ or http://graal.ens-lyon.fr/MUMPS.

Operto, S., Virieux, J., Dessa, J. X., and Pascal, G. (2006). Crustal imaging from multifold ocean bottom seismometers data 
by frequency-domain full-waveform tomography: application to the eastern Nankai trough. Journal of Geophysical Research, 111(B09306):doi:10.1029/2005JB003835.

Pratt, R. G., Shin, C., and Hicks, G. J. (1998). Gauss-Newton and full Newton methods in frequency-space seismic waveform inversion. Geophysical Journal International, 133:341-362.

Pratt, R. G., Song, Z. M., and Warner, M. (1996). Two-dimensional velocity models from wide-angle seismic data by wavefield inversion. Geophysical Journal International, 124:323-340.

Pratt, R. G. and Worthington, M. H. (1990). Inverse theory applied to multi-source cross-hole tomography. Part I: acoustic wave-equation method. Geophysical Prospecting, 38:287-310.

Ravaut, C., Operto, S., Improta, L., Virieux, J., Herrero, A., and dell'Aversana, P. (2004). Multi-scale imaging of complex structures from multi-fold wide-aperture seismic data by frequency-domain full-wavefield inversions: application to a thrust belt. Geophysical Journal International, 159:1032-1056.

Remaki, M. (2000). A new finite volume scheme for solving Maxwell's system. COMPEL, 19(3):913-931.

Robertsson, J. O. A. (1996). A numerical free-surface condition for elastic/viscoelastic finite-difference modeling in the presence of topography. Geophysics, 61:1921-1934.

Saenger, E. H. and Bohlen, T. (2004). Finite-difference modelling of viscoelastic and anisotropic wave propagation using the rotated staggered grid. Geophysics, 69:583-591.

Saenger, E. H., Gold, N., and Shapiro, S. A. (2000). Modeling the propagation of elastic waves using a modified finite-difference grid. Wave motion, 31:77-92.

Stekl, I. and Pratt, R. G. (1998). Accurate viscoelastic modeling by frequency-domain finite difference using rotated operators. Geophysics, 63:1779-1794.

Vilotte, J., Festa, G., and Madariaga, R. (2005). Spectral element simulations of rupture dynamics along kinked faults. In EOS Transactions, volume 86. American Geophysical Union, San Francisco, USA.

Virieux, J. (1986a). P-SV wave propagation in heterogeneous media, velocity-stress finite difference method. Geophysics, 51:889-901.

Virieux, J. (1986b). Séismes: rupture et onde (volumes I et II). PhD thesis, Université Paris VII.

Yee, K. S. (1966). Numerical solution of initial boundary value problems involving Maxwell's equations in isotropic media. IEEE Trans. Antennas and Propagation, 14:302-307.

Yoshimura, C., Bielak, J., Hisada, Y., and Fernandez, A. (2003). Domain Reduction Method for Three-Dimensional Earthquake Modeling in Localized Regions, Part II: Verification and Applications. Bulletin of the Seismological Society of America, 93(2):825-841.

\section{APPENDIX A: EQUIVALENCE FV $P_{0}$ AND $O\left(\Delta X^{2}\right)$ FD IN REGULAR SQUARE GRIDS}

The proposed FV method can be developed in any polygonal mesh structure. In a mesh of regular squares, the FV formulation is equivalent to the second-order FD Cartesian stencil, as we now show. Let us start with the first-order discrete system in velocity and stress :

$$
\begin{aligned}
\omega^{2} \vec{V}_{k} & =\frac{\iota \omega}{A_{k} \rho_{k}} \sum_{j \in \partial K_{k}} l_{k j}\left(n_{k j_{x}} N_{x} s_{x_{j}}+n_{k j_{z}} N_{z} s_{z_{j}}\right) \frac{\vec{T}_{j}}{2}+\iota \omega \vec{F}_{k} \\
\omega^{2} \vec{T}_{k} & =\frac{\iota \omega \Lambda_{k}^{-1}}{A_{k}} \sum_{j \in \partial K_{k}} l_{k j}\left(n_{k j_{x}} M_{x} s_{x_{j}}^{\prime}+n_{k j_{z}} M_{z} s_{z_{j}}^{\prime}\right) \frac{\vec{V}_{j}}{2}+\omega^{2} \vec{T}_{k}{ }_{k},
\end{aligned}
$$

where the cell numbering is denoted by the global index $k$. We modify the numbering of the cells towards a double index system (i,j) that is more suitable for a mesh of squares (Figure 22) where the cell $(i, j)$ has four neighbours located in positions $(i, j-1),(i-1, j),(i+1, j)$ and $(i, j+1)$. The quantity $h$ denotes the constant step length of the grid. The system (A1) can now be written as a vectorial expression with two subscript indices:

$$
\begin{aligned}
\omega^{2} \overrightarrow{V_{i, j}} & =\frac{\iota \omega}{h^{2} \rho_{i, j}}\left(-h N_{x} s_{x_{i, j-1}} \frac{\overrightarrow{T_{i, j-1}}}{2}+h N_{x} s_{x_{i, j+1}} \frac{\overrightarrow{T_{i, j+1}}}{2}-h N_{z} s_{z_{i-1, j}} \frac{\overrightarrow{T_{i-1, j}}}{2}\right. \\
& \left.+h N_{z} s_{z_{i+1, j}} \frac{\overrightarrow{T_{i+1, j}}}{2}\right)+\iota \omega \overrightarrow{F_{i, j}} \\
\omega^{2} \overrightarrow{T_{i, j}} & =\frac{\iota \omega \Lambda_{i, j}^{-1}}{h^{2}}\left(-h M_{x} s_{x_{i, j-1}}^{\prime} \frac{\overrightarrow{V_{i, j-1}}}{2}+h M_{x} s_{x_{i, j+1}}^{\prime} \frac{\overrightarrow{V_{i, j+1}}}{2}-h M_{z} s_{z_{i-1, j}} \frac{\overrightarrow{V_{i-1, j}}}{2}\right. \\
& \left.+h M_{z} s_{z_{i+1, j}}^{\prime} \frac{\overrightarrow{V_{i+1, j}}}{2}\right)+\omega^{2} \vec{T}_{i, j}^{0},
\end{aligned}
$$




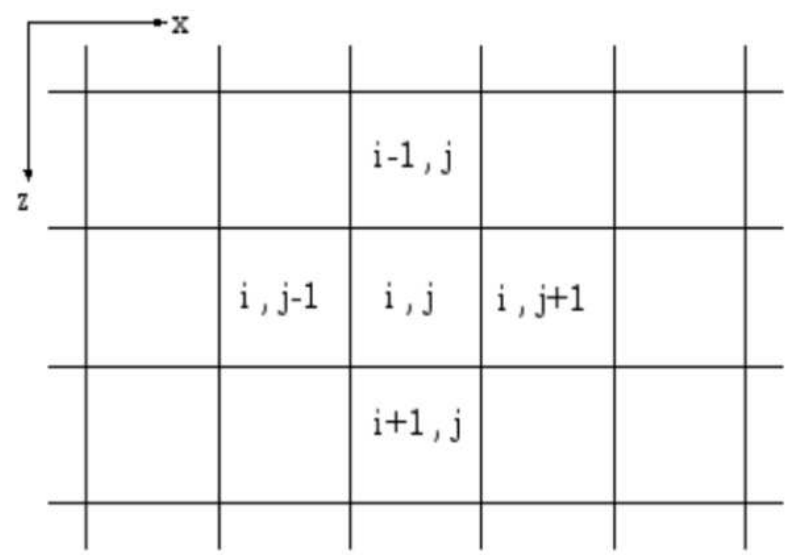

Figure A1. The regular Cartesian mesh for the development of the FV method equivalent to $O\left(\Delta x^{2}\right)$ order FD.

which gives the following system of equations for scalar quantities :

$$
\begin{aligned}
\omega^{2} V_{x_{i, j}} & =\frac{\iota \omega}{2 h \rho_{i, j}}\left(-s_{x_{i, j-1}} T_{1_{i, j-1}}-s_{x_{i, j}-1} T_{2_{i, j-1}}+s_{x_{i, j+1}} T_{1_{i, j}+1}+s_{x_{i, j+1}} T_{2_{i, j}+1}\right. \\
& \left.-s_{z_{i-1, j}} T_{3_{i-1, j}}+s_{z_{i+1, j}} T_{3_{i+1, j}}\right)+\iota \omega F_{x_{i, j}} \\
\omega^{2} V_{z_{i, j}} & =\frac{\iota \omega}{2 h \rho_{i, j}}\left(-s_{x_{i, j-1}} T_{3_{i, j-1}}+s_{x_{i, j+1}} T_{3_{i, j+1}}-s_{z_{i-1, j}} T_{1_{i-1, j}}+s_{z_{i-1, j}} T_{2_{i-1, j}}\right. \\
& \left.+s_{z_{i+1, j}} T_{1_{i+1, j}}-s_{z_{i+1, j}} T_{2_{i+1, j}}\right)+\iota \omega F_{z_{i, j}} \\
\omega^{2} T_{1_{i, j}} & =\frac{\iota \omega\left(\lambda_{i, j}+\mu_{i, j}\right)}{2 h}\left(-s_{x_{i, j-1}}^{\prime} V_{x_{i, j-1}}+s_{x_{i, j+1}}^{\prime} V_{x_{i, j+1}}-s_{z_{i-1, j}}^{\prime} V_{z_{i-1, j}}+s_{z_{i+1, j}}^{\prime} V_{z_{i+1, j}}\right)+\omega^{2} T_{1_{i, j}}^{0} \\
\omega^{2} T_{2_{i, j}} & =\frac{\iota \omega \mu_{i, j}}{2 h}\left(-s_{x_{i, j-1}}^{\prime} V_{x_{i, j-1}}+s_{x_{i, j+1}}^{\prime} V_{x_{i, j+1}}+s_{z_{i-1, j}}^{\prime} V_{z_{i-1, j}}-s_{z_{i+1, j}}^{\prime} V_{z_{i+1, j}}\right)+\omega^{2} T_{2_{i, j}}^{0} \\
\omega^{2} T_{3_{i, j}} & =\frac{\iota \omega \mu_{i, j}}{2 h}\left(-s_{x_{i, j-1}}^{\prime} V_{z_{i, j-1}}+s_{x_{i, j+1}}^{\prime} V_{z_{i, j+1}}-s_{z_{i-1, j}}^{\prime} V_{x_{i-1, j}}+s_{z_{i+1, j}}^{\prime} V_{x_{i+1, j}}\right)+\omega^{2} T_{3_{i, j}}^{0},
\end{aligned}
$$

which are equivalent to the system of discrete equations of a full second-order Cartesian FD stencil inside which two staggered sub-systems propagate the seismic wavefield in an uncoupled way.

\section{APPENDIX B: FINITE VOLUME DEVELOPMENT}

The FV method is applied to first-order elastodynamic systems described by equations (2). By introducing the projector matrices $M_{x}, N_{x}$, $M_{z}$ and $N_{x}$ defined by:

$$
\begin{aligned}
& N_{x}=M_{x}^{t}=\left[\begin{array}{lll}
1 & 1 & 0 \\
0 & 0 & 1
\end{array}\right] \\
& N_{z}=M_{z}^{t}=\left[\begin{array}{ccc}
0 & 0 & 1 \\
1 & -1 & 0
\end{array}\right]
\end{aligned}
$$

and the diagonal matrix $\Lambda=\operatorname{diag}\left(\frac{1}{\lambda+\mu}, \frac{1}{\mu}, \frac{1}{\mu}\right)$, system (2) can be written in a vectorial form with a divergence expression:

$$
\begin{aligned}
-\iota \omega \rho \vec{V} & =\overrightarrow{\operatorname{div}\left(s_{x} N_{x} \vec{T}, s_{z} N_{z} \vec{T}\right)}-\frac{\partial s_{x} N_{x}}{\partial x} \vec{T}-\frac{\partial s_{z} N_{z}}{\partial z} \vec{T}+\rho \vec{F} \\
-\iota \omega \Lambda \vec{T} & =\overrightarrow{\operatorname{div}\left(s_{x}^{\prime} M_{x} \vec{V}, s_{z}^{\prime} M_{z} \vec{V}\right)}-\frac{\partial s_{x}^{\prime} M_{x}}{\partial x} \vec{V}-\frac{\partial s_{z}^{\prime} M_{z}}{\partial z} \vec{V}-\iota \omega \Lambda \overrightarrow{T^{0}}
\end{aligned}
$$

We then introduce vectorial forms: $\vec{G}(\vec{T})=\left(s_{x} N_{x} \vec{T}, s_{z} N_{z} \vec{T}\right)$ and $\vec{H}(\vec{V})=\left(s_{x}^{\prime} M_{x} \vec{V}, s_{z}^{\prime} M_{z} \vec{V}\right)$, and apply a surface integration over a control cell identified by the index $i$.

$$
\begin{aligned}
\int_{K_{i}}-\iota \omega \rho \vec{V} d S & =\int_{K_{i}} \overrightarrow{\operatorname{div}(\vec{G}(\vec{T}))} d S-\int_{K_{i}} \frac{\partial s_{x} N_{x}}{\partial x} \vec{T} d S-\int_{K_{i}} \frac{\partial s_{z} N_{z}}{\partial z} \vec{T} d S+\int_{K_{i}} \rho \vec{F} d S \\
\int_{K_{i}}-\iota \omega \Lambda \vec{T} d S & =\int_{K_{i}} \overrightarrow{\operatorname{div}(\vec{H}(\vec{V}))} d S-\int_{K_{i}} \frac{\partial s_{x}^{\prime} M_{x}}{\partial x} \vec{V} d S-\int_{K_{i}} \frac{\partial s_{z}^{\prime} M_{z}}{\partial z} \vec{V} d S-\int_{K_{i}} \iota \omega \Lambda \vec{T}^{0} d S
\end{aligned}
$$


Through Green's theorem, the surface integration of divergence terms allows flux integrals to appear:

$$
\begin{aligned}
\int_{K_{i}}-\iota \omega \rho \vec{V} d S & =\int_{\partial K_{i}} \vec{G}(\vec{T}) \vec{n} d L-\int_{K_{i}} \frac{\partial s_{x} N_{x}}{\partial x} \vec{T} d S-\int_{K_{i}} \frac{\partial s_{z} N_{z}}{\partial z} \vec{T} d S+\int_{K_{i}} \rho \vec{F} d S \\
\int_{K_{i}}-\iota \omega \Lambda \vec{T} d S & =\int_{\partial K_{i}} \vec{H}(\vec{V}) \vec{n} d L-\int_{K_{i}} \frac{\partial s_{x}^{\prime} M_{x}}{\partial x} \vec{V} d S-\int_{K_{i}} \frac{\partial s_{z}^{\prime} M_{z}}{\partial z} \vec{V} d S-\int_{K_{i}} \iota \omega \Lambda \overrightarrow{T^{0}} d S
\end{aligned}
$$

where $\partial K_{i}$ is the boundaries of cell $K_{i}$ and $\vec{n}$ is the external normal vector of $\partial K_{i}$.

We end up with the already explained discrete system written in a vectorial form. The partial derivatives of all of the PML functions are cancelled by the $P_{0}$ assumption:

$$
\begin{aligned}
-\iota \omega A_{i} \rho_{i} \vec{V}_{i} & =\sum_{j \in \partial K_{i}} l_{i j} G_{i j}+A_{i} \rho_{i} \vec{F}_{i} \\
-\iota \omega A_{i} \Lambda_{i} \vec{T}_{i} & =\sum_{j \in \partial K_{i}} l_{i j} H_{i j}-\iota \omega A_{i} \Lambda_{i} \vec{T}^{0}
\end{aligned}
$$

The integration of property (4) and flux formulation (3) into the discrete system (B5) gives the first-order discrete system where the parsimonious strategy can be applied:

$$
\begin{aligned}
\omega^{2} \vec{V}_{i} & =\frac{\iota \omega}{A_{i} \rho_{i}} \sum_{j \in \partial K_{i}} l_{i j}\left(n_{i j_{x}} N_{x} s_{x_{j}}+n_{i j_{z}} N_{z} s_{z_{j}}\right) \frac{\vec{T}_{j}}{2}+\iota \omega \vec{F}_{i} \\
\omega^{2} \vec{T}_{i} & =\frac{\iota \omega \Lambda_{i}^{-1}}{A_{i}} \sum_{j \in \partial K_{i}} l_{i j}\left(n_{i j_{x}} M_{x} s_{x_{j}}^{\prime}+n_{i j_{z}} M_{z} s_{z_{j}}^{\prime}\right) \frac{\vec{V}_{j}}{2}+\omega^{2} \vec{T}_{i}{ }_{i}
\end{aligned}
$$

